\title{
Retrieval of Vegetation Height in Rice Fields using Polarimetric SAR Interferometry with TanDEM-X Data
}

\author{
Juan M. Lopez-Sanchez ${ }^{\mathrm{a}, *}$, Fernando Vicente-Guijalba ${ }^{\mathrm{a}}$, Esra Erten ${ }^{\mathrm{b}}$, Manuel Campos-Taberner ${ }^{\mathrm{c}}$, Francisco \\ Javier Garcia-Haroc \\ ${ }^{a}$ Institute of Computing Research, University of Alicante, P.O. Box 99, E-03080 Alicante, Spain \\ ${ }^{b}$ Faculty of Civil Engineering, Istanbul Technical University, TR-34469 Istanbul, Turkey \\ ${ }^{c}$ Department of Earth Physics and Thermodynamics, Faculty of Physics, Universitat de Valencia, Dr. Moliner, Burjassot 46100, \\ Valencia, Spain
}

\section{Abstract}

This work presents for the first time a demonstration with satellite data of polarimetric SAR interferometry (PolInSAR) applied to the retrieval of vegetation height in rice fields. Three series of dual-pol interferometric SAR data acquired with large baselines $(2-3 \mathrm{~km})$ by the TanDEM-X system during its science phase (AprilSeptember 2015) are exploited. A novel inversion algorithm especially suited for rice fields cultivated in flooded soil is proposed and evaluated. The validation is carried out over three test sites located in geographically different areas: Sevilla (SW Spain), Valencia (E Spain), and Ipsala (W Turkey), in which different rice types are present. Results are obtained during the whole growth cycle and demonstrate that PolInSAR is useful to produce accurate height estimates (RMSE 10-20 cm) when plants are tall enough (taller than $25-40 \mathrm{~cm}$ ), without relying on external reference information.

Keywords: Agriculture, TanDEM-X, Rice, Synthetic Aperture Radar (SAR), PolSAR, Interferometry, PolInSAR, Vegetation height

\section{Introduction}

Vegetation height is an important agronomic trait related with crop type and potential yield. The seasonal estimation of vegetation height at high resolution from satellite synthetic aperture radar (SAR) data would allow monitoring crop growth status and potentially provides support to agricultural monitoring services. Quantitative information about vegetation height becomes a key input to the classification of crop types and biomass estimation, to improve cultivation management practices, such as precision fertilisation (e.g. to minimise the yield pattern variability within each parcel) and to assess damages and yield reduction resulted from diseases, pests, weather disasters and cereal lodging.

Polarimetric SAR interferometry (PolInSAR) is a well-known radar remote sensing technique for providing structural parameters of vegetation covers (Cloude and Papathanassiou, 1998). It works 
combining at least two polarimetric SAR images using interferometry. Two key aspects to be considered for the right performance of this methodology are the effect of temporal decorrelation and the influence of the spatial baseline. On the one hand, temporal decorrelation stands for the degradation in phase quality due to changes occurred in the scene in the time interval between the acquisitions. These changes may be due to the scene itself (growth of plants, phenological changes, etc.) or to weather effects like wind, which causes a movement in the plant elements, or other events (rain, snow, etc.) which change the scene itself by modifying its geometry and its dielectric features. To avoid temporal decorrelation the two images should be acquired simultaneously (also known as single-pass configuration). When it is not possible, i.e. in a repeat-pass configuration, the time interval should be as short as possible in order to reduce the chance of changes in the scene. On the other hand, the spatial baseline is the distance between the positions of the radar sensors when they acquire the images. It determines the sensitivity of the system to the microwave scattering profile of the scene along the vertical coordinate, being more sensitive when the baseline is larger.

The potential of PolInSAR to measure vegetation height has been widely confirmed for forests of different types and latitudes by means of data acquired with airborne sensors (Garestier et al., 2008; Hajnsek et al., 2009; Lee et al., 2013; Papathanassiou and Cloude, 2001; Praks et al., 2007). Airborne data are gathered with time intervals of minutes to hours, and, due to the large height of trees (typically more than $10 \mathrm{~m}$ ), the required spatial baseline is not very large. Regarding the use of satellite data, in the past all SAR sensors provided revisit times which are too long to avoid an excessive temporal decorrelation (11 days for TerraSAR-X and 12 days for Sentinel-1a are the shortest to date) and only a few gathered polarimetric data. This situation changed with the launch of TanDEM-X, a system in which two identical satellites operate in close formation (Krieger et al., 2007). Recently, several authors have reported successful results in forest height retrieval with PolInSAR by exploiting TanDEM-X data (Abdullahi et al., 2016; Kugler et al., 2015, 2014; Lee and Fatoyinbo, 2015).

As for agriculture, to date the only examples of PolInSAR-based retrieval of crop height correspond to data acquired in indoor experiments (Ballester-Berman et al., 2005; Cloude, 2007; Gomez-Dans et al., 2006; Lopez-Sanchez et al., 2007; Sagués et al., 2000) and more recently by airborne sensors (Lopez-Sanchez et al., 2012b; Pichierri, 2016; Pichierri et al., 2016). The spatial baseline provided by TanDEM-X during its first years of operation, 200-300 m, was designed for the generation of a global DEM and is also suitable for forest height estimations, but it is too short for agriculture (Lopez-Sanchez and Ballester-Berman, 2009). Fortunately, during the science phase of this mission, from April to September 2015, baselines of 2-3 km were employed, hence opening the opportunity to test PolInSAR over agricultural crops with satellite data 
for the first time. Erten et al. (2016) have just published a first work with an example of rice height retrieval using a single acquisition at the end of the growth season over an area located in Ipsala (Turkey). The present work is aimed at completing that study and providing a whole validation on the retrieval of rice height by means of PolInSAR with TanDEM-X data by showing its performance over three different test sites, separated geographically and with different rice varieties, and along the whole cultivation campaign, from sowing to maturation stage.

Rice has been chosen as the ideal crop for this first experiment because of its evident socio-economic interest as the main staple food in the world and, moreover, due to its well-studied radar response with TerraSAR-X (Kucuk et al., 2016; Lopez-Sanchez et al., 2011, 2012a, 2015; Yuzugullu et al., 2015). As it will be explained in the next section, the specific characteristics of rice fields (especially the flooded soil) impose some modifications in the usual direct model of the interferometric coherences and the subsequent inversion algorithm. The retrieval algorithm adapted to this type of crop is proposed in this work for the first time.

The text is organised as follows. Section 2 reviews briefly the formulation employed in PolInSAR and describes the height retrieval procedure proposed in this work. Then, Section 3 presents the three test sites, the associated ground campaigns, and the TanDEM-X data that will be employed, as well as the processing steps carried out with them. Section 4 shows the results and compares the estimates with the validation data. Finally, conclusions are drawn in Section 5.

\section{Methodology}

For readability purposes, this section starts with a brief summary of the formulation of the PolInSAR observables (complex coherences) and the model used to describe a scene with vegetation. Then, the inversion algorithm proposed in this paper is detailed.

\subsection{Formulation and direct model}

TanDEM-X provides pairs of dual-pol images, in which the polarisation channels are chosen by the user. In our case the two co-polarised channels were employed, i.e. HH and VV. Consequently, each image can be expressed as a scattering vector $\vec{k}$ with two entries:

$$
\vec{k}_{1}=\left[S_{H H}^{1}+S_{V V}^{1}, S_{H H}^{1}-S_{V V}^{1}\right]^{T} / \sqrt{2}, \quad \text { and } \quad \vec{k}_{2}=\left[S_{H H}^{2}+S_{V V}^{2}, S_{H H}^{2}-S_{V V}^{2}\right]^{T} / \sqrt{2},
$$

where $S_{P P}^{i}$ corresponds to the complex scattering amplitude of the $i$-th image $(i=1,2)$ at the PP channel, with $\mathrm{PP}=\mathrm{HH}$ or $\mathrm{VV}$, and the $T$ superscript denotes transposition. We have expressed the scattering vectors in the Pauli basis as it is usually done in PolInSAR (Cloude and Papathanassiou, 1998). 
In order to form interferograms, both scattering vectors have to be converted into scalars. For this purpose, unitary complex vectors $\vec{w}$ are employed to select certain polarisation combination, yielding:

$$
S_{1}(\vec{w})=\vec{w}^{* T} \cdot \vec{k}_{1}, \quad \text { and } \quad S_{2}(\vec{w})=\vec{w}^{* T} \cdot \vec{k}_{2}
$$

The interferometric combination of both scalars results in the following expression for the complex interferometric coherence $\gamma$ (Cloude and Papathanassiou, 1998; Kugler et al., 2014):

$$
\gamma\left(\kappa_{Z}, \vec{w}\right)=\frac{\vec{w}^{* T}\left[\Omega_{12}\left(\kappa_{Z}\right)\right] \vec{w}}{\sqrt{\left(\vec{w}^{* T}\left[T_{11}\right] \vec{w}\right)\left(\vec{w}^{* T}\left[T_{22}\right] \vec{w}\right)}},
$$

where

$$
\begin{array}{r}
{\left[\Omega_{12}\left(\kappa_{Z}\right)\right]=\left\langle\vec{k}_{1} \cdot \vec{k}_{2}^{* T}\right\rangle} \\
{\left[T_{11}\right]=\left\langle\vec{k}_{1} \cdot \vec{k}_{1}^{* T}\right\rangle} \\
{\left[T_{22}\right]=\left\langle\vec{k}_{2} \cdot \vec{k}_{2}^{* T}\right\rangle}
\end{array}
$$

are the matrices containing polarimetric information $\left(\left[T_{11}\right]\right.$ and $\left[T_{22}\right]$ ) and both polarimetric and interferometric information $\left(\left[\Omega_{12}\left(\kappa_{Z}\right)\right]\right)$, and $\langle\cdot\rangle$ denotes spatial averaging or multilooking. In this expression $\kappa_{Z}$ is the interferometric vertical wavenumber, which depends on the spatial baseline and the incidence angle.

The region on the unit circle defined by the position of the interferometric coherences for all possible $\vec{w}$ is called the coherence region (grey ellipse in Fig. 1) (Flynn et al., 2002) and is exploited to understand the polarimetric interferometric signature of the observed scene. This region serves to quantify the range of variation of the coherence, both in absolute value and phase, as a function of polarisation, hence providing a measure of the sensitivity of the PolInSAR data to the scene properties.

The measured coherences depend on a number of properties of the sensor and the scene. In order to interpret this dependence and to isolate the terms related to the scene parameters to be retrieved, coherence can be expressed as a product of decorrelation terms, with absolute values bounded between 0 and 1 , as follows:

$$
\gamma=\gamma_{\text {temp }} \cdot \gamma_{\text {geom }} \cdot \gamma_{\text {proc }} \cdot \gamma_{\mathrm{SNR}} \cdot \gamma_{\mathrm{BQ}} \cdot \tilde{\gamma}
$$

where the total coherence $\gamma$ and the last term $\tilde{\gamma}$ are complex numbers, and the rest of decorrelation terms are real numbers. All these terms are described next:

- $\gamma_{\text {temp }}$ is the temporal decorrelation due to changes in the scene occurred during the acquisition times of both images. In a bistatic single-pass interferometer this term does not affect, i.e. $\gamma_{\text {temp }}=1$. 


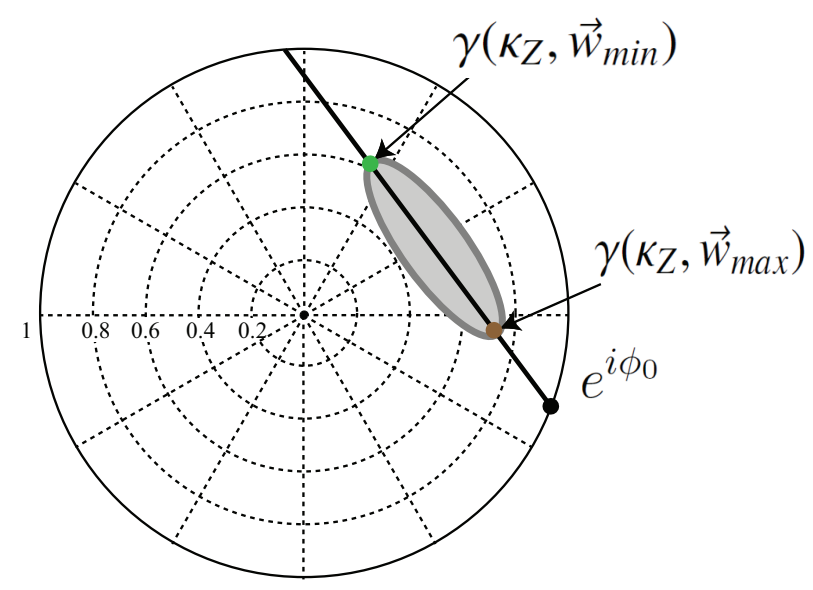

Figure 1: Unit circle on the complex plane with the coherence region (grey ellipse) and the coherences with maximum ground contribution $\gamma\left(\kappa_{Z}, \vec{w}_{\max }\right)$ (brown point) and with minimum ground contribution $\gamma\left(\kappa_{Z}, \vec{w}_{\min }\right)$ (green point). The line corresponds to the standard RVoG model, which crosses the unit circumference at the topographic phase $\phi_{0}$.

- $\gamma_{\text {geom }}$ is the decorrelation due to the spatial baseline, also named as geometric decorrelation, which causes a wavenumber shift, i.e. a change in the band occupied by the range coordinate spectrum of both images (Gatelli et al., 1994). This term can be cancelled by filtering the master and slave images to the common frequency band, as it has been done in this work (see Section 3 for processing details).

- $\gamma_{\text {proc }}$ includes any decorrelation due to the signal processing steps, in which the most important is usually the one due to errors in the coregistration of the images. In our case we consider it is negligible (i.e. $\gamma_{\text {proc }}=1$ ) thanks to the high accuracy of the TanDEM-X products provided in CoSSC format.

- $\gamma_{\mathrm{SNR}}$ denotes the decorrelation due to thermal noise in the sensor, which depends on the signal-tonoise ratio (SNR) at each pixel. We will discuss it in Section 2.2, since it is quite significant for TanDEM-X and this type of scene.

- $\gamma_{\mathrm{BQ}}$ is the loss of coherence due to the quantisation of the data with less bits than in the original raw data. Its effect is extensively discussed by Martone et al. (2015). Attending to the 8:3 block adaptive quantisation employed in the products (at both TanDEM-X and TerraSAR-X images) and the type of scene observed (agricultural crops), the average value of decorrelation is around $3.5 \%$, i.e. $\gamma_{\mathrm{BQ}} \approx 0.965$. This decorrelation term will be compensated for by dividing the measured coherences by this value.

- $\tilde{\gamma}$ is the coherence due to the vertical distribution of scattering properties of the scene, usually named as volume decorrelation $\gamma_{v o l}$ because it is always present when a vegetation volume is in the scene. 
Here we denote it with a tilde, $\tilde{\gamma}$, because it is the coherence that will be modelled according to the scene features and, consequently, is the main term to be estimated from the measured data. This term is explained next.

The estimation of vegetation height, and other biophysical parameters, by means of PolInSAR is carried out by assuming a model of the vegetated scenes. The most widely used model considers the scene is formed by two layers: a vegetation volume and a ground surface. The scattering from the ground is located at a single point in the vertical coordinate $z_{0}$, whereas the scattering from the volume is distributed according to a scattering function $f(z)$.

Starting from this assumption it is possible to express the coherences $\tilde{\gamma}$ that are obtained at different polarimetric channels $\vec{w}$ as a function of the scene properties and the vertical wavenumber $\kappa_{Z}$. The most complete expression for a bistatic system, considering that the response from the ground can be composed of two contributions (surface or direct scattering, and double-bounce scattering) is the following (BallesterBerman and Lopez-Sanchez, 2007, 2011; Kugler et al., 2014; Treuhaft et al., 1996; Treuhaft and Siqueira, 2000):

$$
\tilde{\gamma}\left(\kappa_{Z}, \vec{w}\right)=e^{i \phi_{0}} \frac{\tilde{\gamma}_{V}+m_{D}(\vec{w})+\frac{\sin k_{z} h_{v}}{k_{z} h_{v}} m_{D B}(\vec{w})}{1+m_{D}(\vec{w})+m_{D B}(\vec{w})}
$$

where $\phi_{0}=\kappa_{Z} z_{0}$ is the interferometric phase corresponding to the ground surface; $m_{D}(\vec{w})$ and $m_{D B}(\vec{w})$ are the ground-to-volume backscatter ratios corresponding to the direct $D$ and double-bounce $D B$ contributions, respectively; and $h_{v}$ is the vegetation height (i.e. the depth of the vegetation volume). The first term in the numerator, $\tilde{\gamma}_{V}$, is the coherence that would produce the volume alone (without the presence of the ground), which can be expressed as a function of $f(z)$ as:

$$
\tilde{\gamma}_{V}=\frac{\int_{0}^{h_{v}} f(z) e^{i K_{Z} z} d z}{\int_{0}^{h_{v}} f(z) d z}
$$

A note of caution is necessary for equation (8). The $\sin (x) / x$ term that appears before the doublebounce ground-to-volume ratio in the numerator is an extra decorrelation term present whenever a bistatic configuration is used. It is important to clarify that the argument of this term is $k_{z} h_{v}$, not $\kappa_{Z} h_{v}$ as was wrongly stated in (Kugler et al., 2014). The $k_{z}$ wavenumber is defined as (see Ballester-Berman and Lopez-Sanchez (2007, 2011); Treuhaft and Siqueira (2000) for details):

$$
k_{z}=\kappa_{Z} \sin ^{2} \theta_{0} .
$$

The scattering function $f(z)$ that appears in (9) can be expressed in different ways according to different models or approximations of the scattering properties of the vegetation volume. The most 
common expression is an exponential decay which corresponds to a homogeneous volume characterised by a constant extinction coefficient. It is well known that in agricultural crops the attenuation produced by the propagation of the waves through the vegetation volume depends on the polarisation of the waves, being normally larger for vertical polarisation than for horizontal polarisation due to the predominant vertical orientation of the plant elements. In such a case, the formulation that takes this into account leads to the so-called oriented volume over ground (OVoG) model, in which two extinction coefficients appear (i.e. vertical and horizontal). However, when this dependence on polarisation is not strong, one can use a simpler model named random volume over ground ( $\mathrm{RVoG})$ in which extinction is assumed to not depend on polarisation and, consequently, a single extinction coefficient is used for all polarimetric channels. The RVoG model is the most frequent in forest studies (Hajnsek et al., 2009; Kugler et al., 2014; Papathanassiou and Cloude, 2001). In our case, because of the reduced dimensionality of the observation space or input data (TanDEM-X data are dual-pol, not fully polarimetric), we need to decrease the number of model parameters to design a feasible inversion strategy. Therefore, we will assume the RVoG model for the inversion. In other words, we have resorted to ignore differential extinction in the formulation to keep the problem invertible. Obviously, this assumption constitutes a source of errors. However, whenever differential extinction is not strong it is a valid assumption, and the RVoG model can be inverted properly, as it is also suggested by Cloude (2009). The same strategy (using RVoG instead of OVoG regardless of the differential extinction present in the scene) has been tested recently by Pichierri (2016), who obtained good height estimates for maize at C-band and for wheat at C- and X-band.

In the case of rice fields, the most common agronomic practice consists of keeping fields flooded during the entire rice growing period. Thus soil background behaves mostly like a mirror, hence producing a very low backscattering. Therefore, the dominant backscattering contribution from the ground is expected to be the double-bounce produced by the interaction between stems and flooded soil. Consequently, equation (8) can be simplified by neglecting the direct contribution from the ground $\left(m_{D} \approx 0\right)$, yielding:

$$
\tilde{\gamma}\left(\kappa_{Z}, \vec{w}\right)=e^{i \phi_{0}} \frac{\tilde{\gamma}_{V}+\frac{\sin k_{z} h_{v}}{k_{z} h_{v}} m_{D B}(\vec{w})}{1+m_{D B}(\vec{w})}
$$

With all these assumptions, the only dependence of coherence (11) on the polarimetric channels comes from the ground-to-volume ratio $m_{D B}(\vec{w})$. This dependence makes the possible coherences (predicted by the RVoG model) to lie along a line on the complex plane (see Fig. 1), which is a geometrical feature exploited by most of the inversion algorithms based on the RVoG model (Cloude and Papathanassiou, 2003; Papathanassiou and Cloude, 2001). 


\subsection{Inversion algorithm}

\subsubsection{Inversion strategy}

There exist different ways to invert vegetation height (and the rest of model parameters) from PolInSAR data according to the previous expressions. In this work we base the inversion procedure on the algorithm proposed by Kugler et al. (2014) also for TanDEM-X data. The interested reader is referred to this study for further details of the algorithm. However, due to the particular properties of rice scenes (i.e. dominance of the double-bounce ground contribution) the algorithm has been adapted, and all details are described here. The proposed algorithm is sketched in Fig. 2. The main steps are:

1. Line fit to a set of coherences or coherence region, and estimation of the two coherences with maximum and minimum ground contributions: $\gamma\left(\kappa_{Z}, \vec{w}_{\max }\right)$ and $\gamma\left(\kappa_{Z}, \vec{w}_{\min }\right)$.

2. SNR and BQ correction of the two coherences.

3. Numerical estimation of the model parameters: topographic phase $\phi_{0}$, vegetation height $h_{v}$, extinction $\sigma$ and ground-to-volume ratios.

The first step consists of a line fit to the coherences, which can be carried out in several ways (Cloude, 2009). The original approach published in the literature consists of obtaining a set of coherences by using a discrete set of projection vectors $\vec{w}$. A common choice for the set consists in obtaining the coherences in the Pauli basis, in the linear basis, and the optimum coherences (Cloude and Papathanassiou, 1998), which would yield a set of 6 coherences for this dual-pol case. Then, a least square fit on the complex plane is employed to get the line that best fits the set of coherences (Cloude and Papathanassiou, 2003). Alternatively, the line fit could be obtained by using a maximum likelihood approach with respect to the RVoG model, which was formulated by Ferro-Famil et al. (2009) for quad-pol data. A third option, which is quite simple, consists in generating the border of the coherence region (grey ellipse in Fig. 1) and choosing the coherences with minimum and maximum phase to define the line (Kugler et al., 2014). This means that we choose the coherences (from the coherence region) which are closest to and farthest from the topographic phase $\phi_{0}$. In this work we have used this last approach because its principle also coincides with the objective of selecting the coherences with maximum and minimum ground contribution, i.e. $\gamma\left(\kappa_{Z}, \vec{w}_{\max }\right)$ and $\gamma\left(\kappa_{Z}, \vec{w}_{\min }\right)$, which are, by definition, the ones with phases closest to and farthest from the topographic phase, respectively (see Fig. 1).

Once this pair of coherences is selected, the effect of signal-to-noise ratio (SNR) has to be compensated. This is the main decorrelation contribution (apart from the vegetation volume) for TanDEM-X (Krieger et al., 2007) once range spectral filtering has been applied. SNR is known to affect interferometric products 


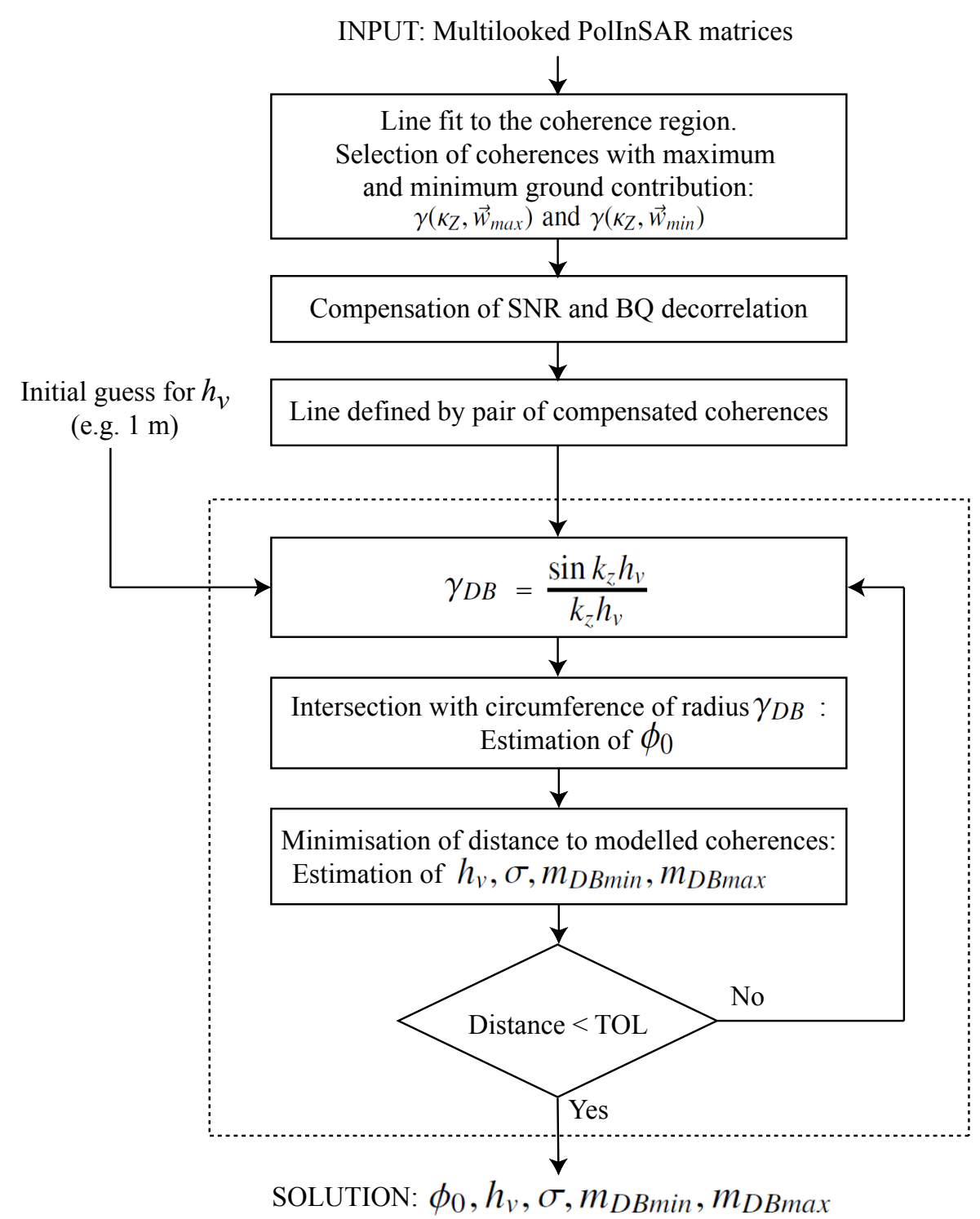

Figure 2: Flow diagram of the proposed inversion algorithm for PolInSAR data and scenes with a dominant double-bounce ground contribution.

according to the following expression (Bamler and Hartl, 1998),

$$
\gamma_{\mathrm{SNR}}=\frac{\mathrm{SNR}}{1+\mathrm{SNR}}
$$

in which the power of signal and noise are considered the same in both images (master and slave). This decorrelation factor is usually ignored in interferometric studies because it only affects areas with low backscatter. Unfortunately, the backscatter level present in SAR images acquired at X-band over rice fields is normally in the range from -25 to $-5 \mathrm{~dB}$, changing along the growing season, and these values are not much higher than the noise level of TanDEM-X data (from -25 to -20 dB approximately). 
The standard TanDEM-X products provide the annotated values of noise equivalent sigma zero (NESZ) patterns for each channel in the form of a set of polynomial coefficients for the range coordinate, with an update every 1.5 to $2 \mathrm{~s}$ in azimuth time, so NESZ can be computed by polynomial evaluation and interpolation for every pixel in the images. The values of NESZ depend on the beam used for the acquisitions (incidence angle), the polarimetric channel (HH and VV), and the satellite (they are different for TerraSAR-X and TanDEM-X). Typical values of NESZ for TanDEM-X are shown in (Kugler et al., 2014).

For each one of the copolar channels, the SNR can be calculated by using the corresponding NESZ value and the backscattering coefficient $\sigma_{0}$, i.e.,

$$
\mathrm{SNR}_{P P}^{i}=\frac{\sigma_{0 P P}^{i}-\mathrm{NESZ}_{P P}^{i}}{\mathrm{NESZ}_{P P}^{i}}
$$

where subscript PP denotes the channel (HH or VV), and superscript $i=1,2$ denotes the image (master or slave) because they are acquired by different satellites (TerraSAR-X or TanDEM-X), hence showing different noise levels.

For illustration purposes, Figure 3 shows the evolution of the measured coherence (after range spectral filtering) and the SNR decorrelation, $\gamma_{\mathrm{SNR}}$, for the two copolar channels, acquired over a rice parcel in Sevilla during the 2015 campaign. The influence of SNR over the total coherence is obvious, especially for the VV channel because it is characterised by a lower backscatter level (hence a lower SNR) for most of the season. It is important to note that this SNR effect in TanDEM-X data acquired over rice fields was already discussed by Lopez-Sanchez et al. (2013), concluding that the total coherence was virtually equal to the SNR term. However, in that reference the spatial baseline was small (the typical height of ambiguity was above $100 \mathrm{~m}$ ) compared to the one available during the science phase (height of ambiguity between 2 and $6 \mathrm{~m}$ ) so the system did not provide sensitivity to the volume decorrelation $\tilde{\gamma}$.

The effect of SNR is compensated by dividing the coherences (at this point the coherences with extreme ground contributions, $\gamma\left(\kappa_{Z}, \vec{w}_{\text {max }}\right)$ and $\left.\gamma\left(\kappa_{Z}, \vec{w}_{\text {min }}\right)\right)$ by $\gamma_{\mathrm{SNR}}$. Therefore, the SNR decorrelation has to be obtained for the specific polarimetric combinations $\vec{w}_{\max }$ and $\vec{w}_{\min }$, so we need to compute the NESZ at these specific channels. This can be done by using the following matrix that corresponds to NESZ at the linear channels, which is diagonal by definition:

$$
\left[N_{i}\right]=\left[\begin{array}{cc}
\mathrm{NESZ}_{H H}^{i} & 0 \\
0 & \mathrm{NESZ}_{V V}^{i}
\end{array}\right]
$$



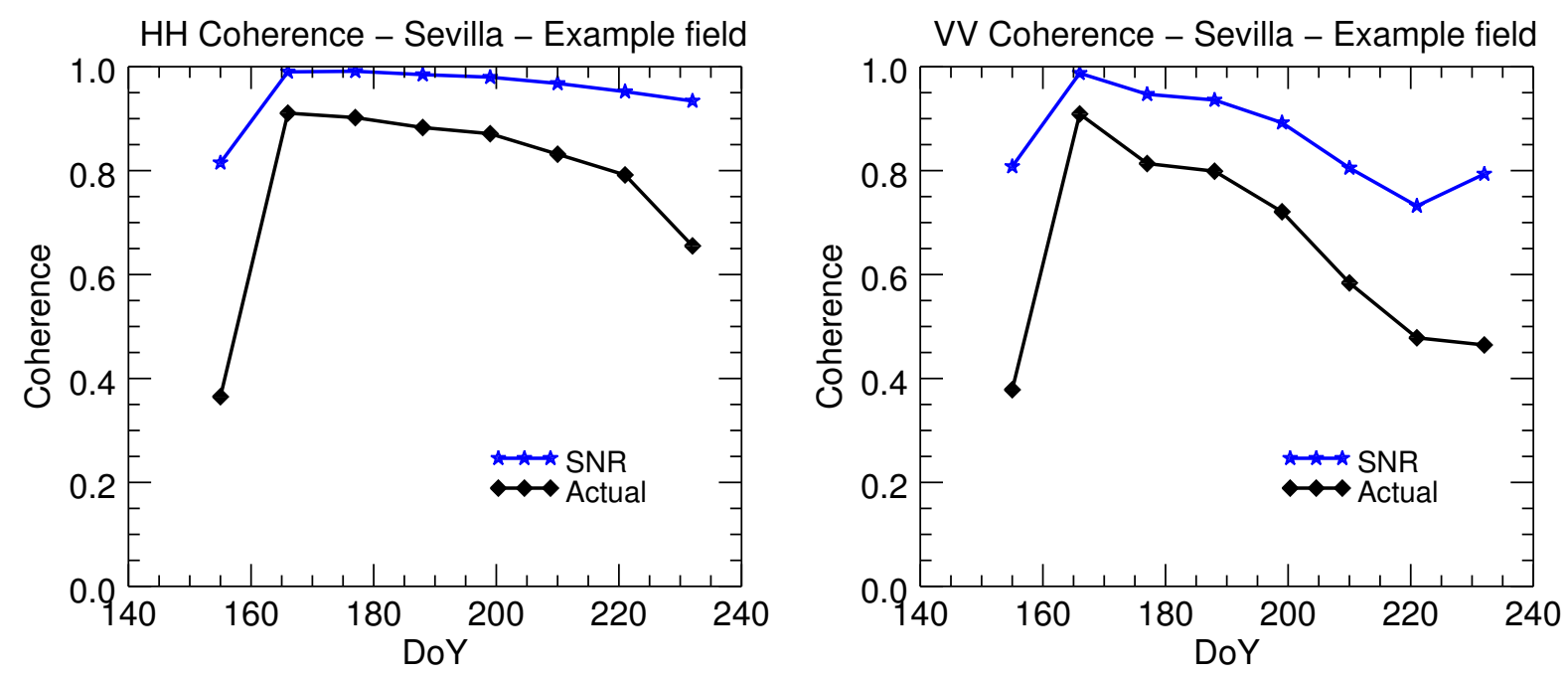

Figure 3: Comparison of the total measured coherence (after range spectral filtering) and the SNR decorrelation term, $\gamma_{\mathrm{SNR}}$, for the two copolar channels obtained for a rice field in Sevilla during the 2015 campaign. Left: HH. Right VV.

and the SNR decorrelation is:

where

$$
\left[U_{2}\right]=\frac{1}{\sqrt{2}}\left[\begin{array}{cc}
1 & 1 \\
1 & -1
\end{array}\right] .
$$

From $\left[N_{i}^{P}\right]$ the noise power at a particular polarisation $\vec{w}$ combination is obtained as:

$$
N_{i}(\vec{w})=\vec{w}^{* T}\left[N_{i}^{P}\right] \vec{w},
$$

and the backscattering coefficient (degraded by additive noise) is obtained as usual:

$$
\sigma_{0 i}(\vec{w})=\vec{w}^{* T}\left[T_{i i}\right] \vec{w} .
$$

Finally the SNR at each image results in:

$$
\operatorname{SNR}_{i}(\vec{w})=\frac{\sigma_{0 i}(\vec{w})-N_{i}(\vec{w})}{N_{i}(\vec{w})},
$$

$$
\gamma_{\mathrm{SNR}}(\vec{w})=\sqrt{\left(\frac{\mathrm{SNR}_{1}(\vec{w})}{1+\mathrm{SNR}_{1}(\vec{w})}\right) \cdot\left(\frac{\mathrm{SNR}_{2}(\vec{w})}{1+\mathrm{SNR}_{2}(\vec{w})}\right)}
$$


After the SNR compensation, both coherences are also corrected for the quantisation effects, which is carried out again as a division by the theoretical $\gamma_{\mathrm{BQ}}$.

Once all these corrections have been applied, we consider the volume term $\tilde{\gamma}$ to be the only feature present in the coherences and the inversion of the direct model of the scene (i.e. estimation of all model parameters) is carried out following the procedure explained here. The proposed method is essentially the same as the one proposed in (Cloude, 2009; Kugler et al., 2014), but there are important changes due to the specific properties of rice scenes, namely: the dominant contribution from the ground is the double-bounce, so the expression to be inverted is (11), and all polarimetric channels present some contribution from the ground (i.e. $m_{D B} \neq 0$ for all $\vec{w}$ ).

The estimation is based on the minimisation of the distance between the measured coherences $\gamma$ and the modelled ones $\tilde{\gamma}$ :

$$
\min _{\phi_{0}, h_{v}, \sigma, m_{D B \min }, m_{D B \max }}\left\|\begin{array}{l}
\gamma\left(\kappa_{Z}, \vec{w}_{\max }\right)-\tilde{\gamma}\left(\kappa_{Z}, \phi_{0}, h_{v}, \sigma, m_{D B \max }\right) \\
\gamma\left(\kappa_{Z}, \vec{w}_{\min }\right)-\tilde{\gamma}\left(\kappa_{Z}, \phi_{0}, h_{v}, \sigma, m_{D B \min }\right)
\end{array}\right\|
$$

Since there are 5 model parameters (unknowns) and only 4 real observables (two complex coherences), we face an underdetermined system to solve. Fortunately, the ground phase $\phi_{0}$ can be obtained by applying a geometrical solution (line fit) and then the remaining four parameters can be found numerically by minimising (21).

The phase $\phi_{0}$ corresponding to the ground topography is usually obtained by the intersection of the line defined by the two coherences, $\gamma\left(\kappa_{Z}, \vec{w}_{\max }\right)$ and $\gamma\left(\kappa_{Z}, \vec{w}_{\min }\right)$, and the unit circumference, moving from $\gamma\left(\kappa_{Z}, \vec{w}_{\min }\right)$ to $\gamma\left(\kappa_{Z}, \vec{w}_{\text {max }}\right)$ (see Figure 1). However, the extra decorrelation term $\gamma_{D B}=\sin k_{z} h_{v} / k_{z} h_{v}$ in the numerator of (11) makes the coherence of pure ground contribution $\left(m_{D B} \rightarrow \infty\right)$ not to be equal to 1 (i.e. it would lie on the unit circumference) but to be equal to $\gamma_{D B}$, so we have to find the crossing of the line with the circumference of radius equal to $\gamma_{D B}$. The main consequence for the inversion is that the topographic phase is shifted with respect to the point at which the line crosses the unit circumference, as it can be observed in Figure 4 (see Ballester-Berman and Lopez-Sanchez (2011) for more details).

Since $\gamma_{D B}$ depends on the vegetation height $h_{v}$, which is one of the unknowns, the topographic phase cannot be estimated directly from any intersection between line and circumference, so an iterative numerical procedure is adopted. As initial guess we consider an average value for the vegetation height (e.g. $\left.h_{v}=1 \mathrm{~m}\right)$. With that value we obtain the corresponding $\gamma_{D B}$ and find the intersection of the line with the circumference of radius $\gamma_{D B}$, which provides an initial value for $\phi_{0}$. With that $\phi_{0}$ value we find the set of $h_{v}, \sigma, m_{D B m a x}$ and $m_{D B m i n}$ which provides the minimum distance between the modelled and the measured coherences. Then, the new value of $h_{v}$ is used to update $\gamma_{D B}$ and hence the topographic phase $\phi_{0}$, and the minimisation is 


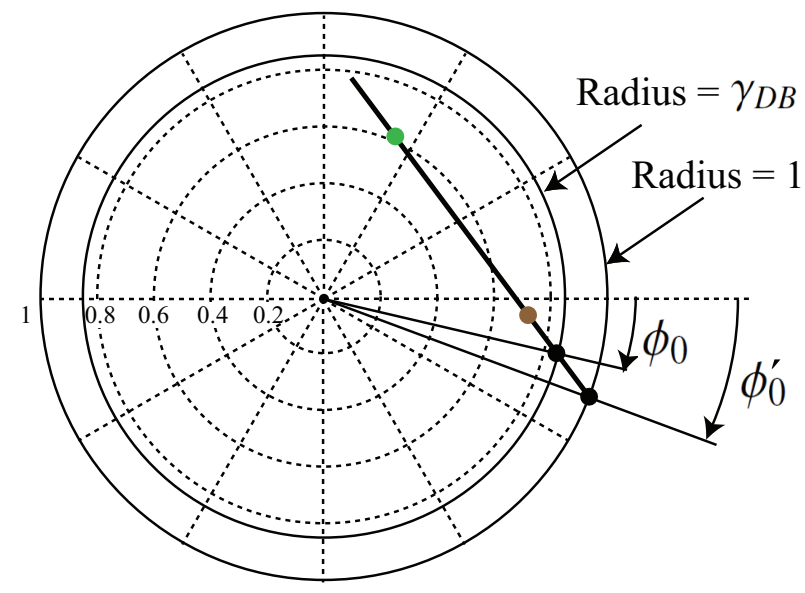

Figure 4: Unit circle on the complex plane with the coherences and the line corresponding to the RVoG model in equation (11). $\gamma_{D B}$ is the decorrelation term due to the dominance of the double-bounce contribution at the ground. The true topographic phase $\phi_{0}$ is defined by the crossing of the line with the circumference of radius $\gamma_{D B}$, which is different from the phase $\phi_{0}^{\prime}$ that would have been obtained by the crossing with the unit circumference.

carried out again. The iteration continues until convergence to a solution with minimum distance between model and observations.

\subsubsection{Assessment of the numerical inversion}

The possible error sources of this technique are related mainly with three different aspects. The first question is how well the direct model represents the scene. Scenes not properly represented by the RVoG model will provide PolInSAR observations (coherences) not fitting the expressions studied here and, consequently, the retrieval of model parameters will produce wrong or meaningless values. The main causes of mismatch between the RVoG model and rice scenes are the presence of non-exponential scattering profiles (e.g. due to a heterogeneous vegetation volume, along the vertical coordinate, caused by the presence of different plant elements at different heights), the effect of differential extinction (i.e. vertically polarised waves are expected to be more attenuated than horizontally polarised ones due to the dominant orientation of stalks and tillers), and a non-negligible direct surface component from the ground, i.e. (8) could not be simplified as (11). The second aspect producing errors in the retrieval procedure is related to all system and data processing aspects, including the effect of baseline and incidence angle on the sensitivity of this technique to the scene properties, but also the presence of speckle, the estimation of coherences using multi-looking, the compensation of SNR and BQ decorrelation, etc. Finally, the third error source is the numerical inversion itself (i.e. the algorithm depicted inside the dashed box in Fig. 2). In this subsection we provide a numerical assessment of this last aspect. Regarding the other error sources, we 
will discuss them further in Section 4.

In single-baseline PolInSAR it is well known that the retrieval of the RVoG parameters does not provide a single solution, but a value within a range of possible solutions for which the minimisation in (21) is satisfied. Different combinations of model parameters (topographic phase, vegetation height, extinction, and ground-to-volume ratios) produce very similar model outputs, so the numerical minimisation in (21) can fall in local minima or simply provide an arbitrary solution depending on the initial guess of these model parameters.

In order to assess the feasibility of the proposed numerical inversion (dashed box in Fig. 2), with focus on estimation of vegetation height, a simulation experiment was carried out. The theoretical coherences $\left(\gamma\left(\kappa_{Z}, \vec{w}_{\max }\right)\right.$ and $\left.\gamma\left(\kappa_{Z}, \vec{w}_{\min }\right)\right)$ provided by the forward model (11) were generated for a wide set of scene parameters. More specifically, vegetation height was set from $5 \mathrm{~cm}$ to $1.50 \mathrm{~m}$, with a $5 \mathrm{~cm}$ sequential increment. For each height in the set, 500 scenes were simulated with values of extinction $(\sigma)$ and groundto-volume ratios $\left(m_{D B \min }, m_{D B \max }\right)$ randomly generated (using a uniform distribution), in the following ranges: 1 to $7 \mathrm{~dB} / \mathrm{m}$ for extinction, and -10 to $10 \mathrm{~dB}$ for the ground-to-volume ratios. The topographic phase $\phi_{0}$ was fixed to 20 degrees, and also incidence angle and $\kappa_{Z}$ remained constant $(25$ degrees and $2 \mathrm{rad} / \mathrm{m}$, respectively). Then, the model was inverted many times for each scene by employing different sets of initial guesses. In this experiment we applied the inversion 500 times for each scene, and the initial guesses were also generated randomly within the following intervals: 0 to $2 \mathrm{~m}$ for height, 0 to $10 \mathrm{~dB} / \mathrm{m}$ for extinction, and -10 to $10 \mathrm{~dB}$ for the ground-to-volume ratios. Finally, the heights retrieved (250.000 in total) for each simulated height were analysed.

Figure 5 shows the average and standard deviation of the retrieved heights for all simulated cases and considering all initial guesses. There is not any noticeable bias produced by the inversion approach. As for the expected standard deviation, i.e. the variability of the results produced by the inversion itself, they are in the same order $(8-15 \mathrm{~cm})$ as the results that will be obtained with real data (see Section 4$)$. It must be noted that some of the combinations of input model parameters for the simulations and some initial guesses could be very unrealistic, but we have preferred not to remove them in the analysis. In the same vein, the retrieved values could be further analysed and filtered by considering the final distance from the modelled coherences (i.e. the norm in eq. 21). For instance, we could keep only the results with the minimum distances, or a percentage of them. However, we preferred not to explore further these options and then focus the rest of the work in the validation with real data. 


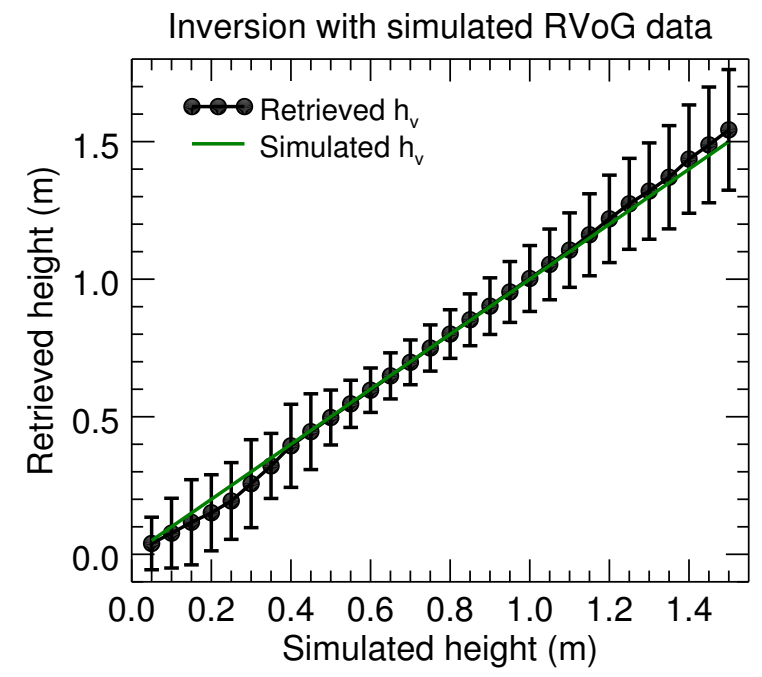

Figure 5: Numerical assessment of the inversion algorithm: vegetation height estimates (mean and standard deviation) for each simulated value in the range $0.05-1.5 \mathrm{~m}$.

\section{Test sites and data sets}

\subsection{Test sites and ground campaign data}

\subsubsection{Sevilla, Spain}

The test site consists of an area of $30 \mathrm{~km} \times 30 \mathrm{~km}$ in the mouth of the Guadalquivir river, Sevilla, SW of Spain $(37.1 \mathrm{~N}, 6.15 \mathrm{~W})$, where rice is cultivated annually from May to October, approximately.

General rice species in this area is Oryza sativa $L$. The specific variety cultivated in the monitored fields corresponds to a long grain type named puntal, quite common in Spain and other similar temperate regions.

In this specific location, sowing is carried out by spreading seeds randomly from an airplane over the fields, which are already flooded at that time. Then, farming practises in this area ensure the presence of a water layer on the ground during the whole cultivation period, hence the ground is always flooded and will be considered as a water surface from the radar point of view. Finally, the cultivation campaign lasts about 135-150 days.

Since 2008, the local association of rice farmers (Federacion de Arroceros de Sevilla) has collected detailed ground measurements on a weekly basis. For this research project, centred in the 2015 campaign, four specific parcels, spread over the whole site (Figure 6a), were selected for intensive sampling. The weekly measurements include phenological stage according to the $\mathrm{BBCH}$ (Biologische Bundesanstalt, Bundessortenamt und CHemische Industrie) scale and above-water vegetation height. The following information is known for each parcel: total area (ha), sowing date, surface density of plants (plants $/ \mathrm{m}^{2}$ ) and 


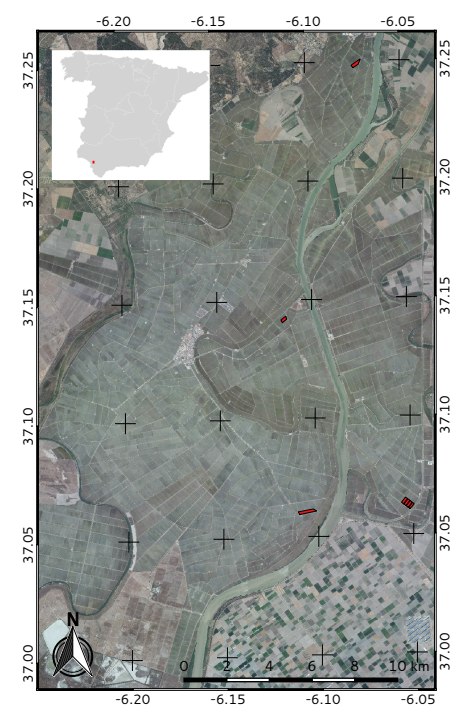

a) Sevilla (Spain)

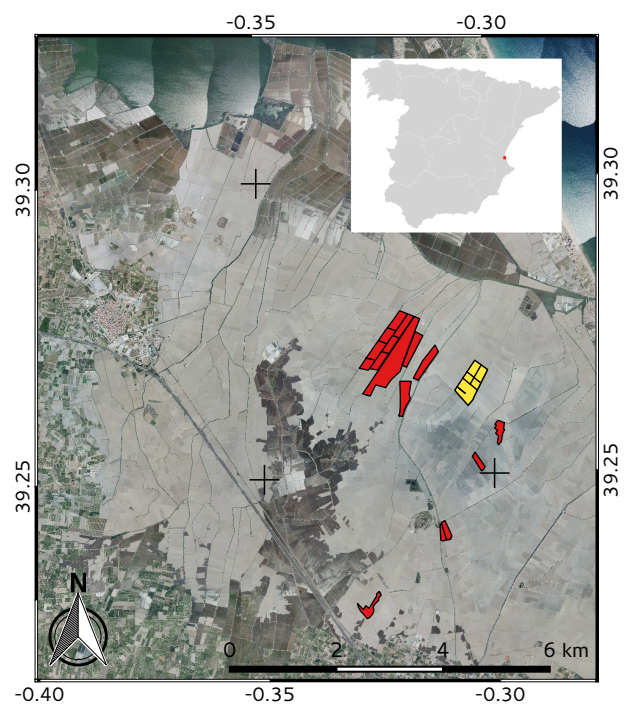

b) Valencia (Spain)

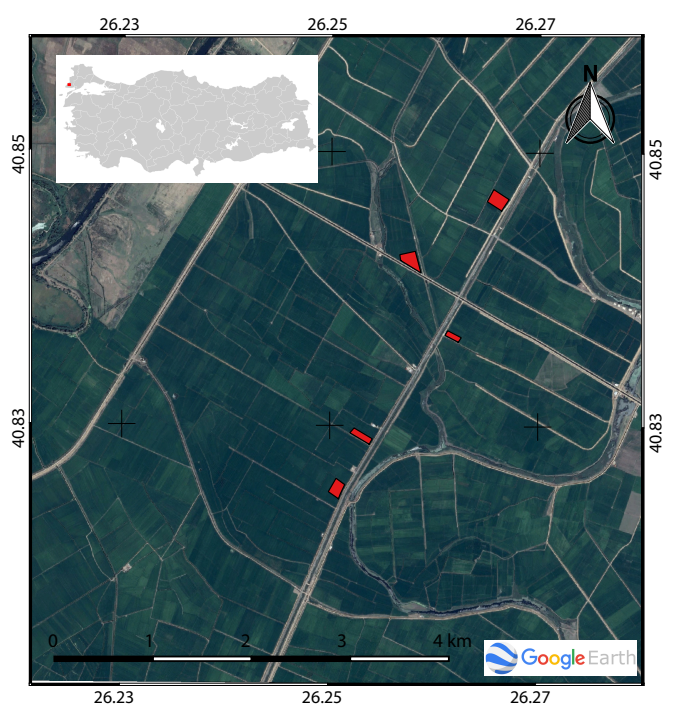

c) Ipsala (Turkey)

Figure 6: Location and orthophotos of the 3 test sites (Sevilla, Valencia and Ipsala). All fields monitored in 2015 are highlighted. In the case of Valencia, the fields coloured in red correspond to Senia type and fields coloured in yellow correspond to Bomba type.

panicles (panicles $/ \mathrm{m}^{2}$ ), harvest date, and final yield $(\mathrm{kg} / \mathrm{ha})$. Particular aspects for some of them have been registered also, such as irrigation conditions, water salinity and presence of plagues. Note that neither sowing nor harvest are simultaneous in all parcels of the site, being around 3 to 4 weeks time span between the first and the last of the monitored parcels in both activities. Finally, there is also climate information provided by the Spanish Government under the Sistema de Informacion Agroclimatica para el Regadio (SIAR), including daily files of temperature, precipitation, humidity and wind. In this region, a rainy season is common at the beginning of autumn every year and did not affect the radar acquisitions available in this study.

This test site has been employed for research purposes in the field of SAR remote sensing, appearing in the following publications, among others: (Kucuk et al., 2016; Lopez-Sanchez et al., 2011, 2012a, 2014, 2015; Yuzugullu et al., 2015).

\subsubsection{Valencia, Spain}

The second study area used in this paper is located in selected farms within the rice district of Sueca $(39.25 \mathrm{~N}, 0.3 \mathrm{~W})$, situated in the south of Valencia, in Eastern Spain (see Figure 6b). This study area belongs to the Albufera Natural Park, in which only rice crop practices are allowed. The area has a typical Mediterranean climate, with an average annual temperature and humidity of $17{ }^{\circ} \mathrm{C}$ and $65 \%$, respectively. The rice district is a homogeneous rice planting area of approximately $10 \mathrm{~km} \times 20 \mathrm{~km}$ and the majority of the paddy rice fields have an extension of $100 \mathrm{~m} \times 200 \mathrm{~m}$. Sowing activities are around May 10-15th 
and fields are managed by keeping them flooded for most of the time during the rice growing period. The maturity stage is reached in early September, and the rice harvest begins in mid-September. The two main rice varieties are Senia and Bomba. The Senia variety has more stacked up stems and leafs, while the Bomba variety has a considerably greater height. Both varieties are under the guarantee granted by the Regulatory Board of Designation of Origin Arròs de València (www.arrozdevalencia.org).

Field campaigns were conducted weekly including the acquisitions of leaf area index (LAI) and phenology according to the BBCH scale within 16 Senia and 5 Bomba parcels (see Figure 6b). Above-water rice heights were taken from previous years by considering the same phenological stage and interpolating for the acquisition dates.

This test site has been employed for research purposes in the field of remote sensing, appearing in the following publications, among others: (Campos-Taberner et al., 2015, 2016a,b).

\subsubsection{Ipsala, Turkey}

The study area is a rice-agricultural site of approximately $6 \mathrm{~km} \times 16 \mathrm{~km}$ situated along the Maritza river, Ipsala, NW of Turkey (N 40.8, 26.2 E). Ipsala site is one of the major rice producing areas in Turkey, providing more than $35 \%$ of the total rice production.

The main rice species present are long-grain Oryza sativa L. types: Baldo and Rocca. Recently, the hybrid types such as Osmancik, Ergene, Serhat and İpsala have been increasingly planted. The seeds are sowed by broadcasting once per year at about the end of May and harvested in late October. Maritza river and its tributaries form the drainage system for irrigated rice fields, which are to be under about $13-15 \mathrm{~cm}$ of water. The fields are continuously flooded up to 30-35 days after germination. Fields are managed according to the different owners' planning. The diversity of irrigation amount and dates has an impact on temporal behaviour of the crops among the fields. Recently, the prevalence of red wild weeds coupled with global warming (long winter without rain) threatens yield in the region.

During the ground measurements conducted by the Directorate of Trakya Agricultural Research Institute, information on total area (ha), water depth, stalk height, height above water, \# stalk $/ \mathrm{m}^{2}$, leaf length, leaf width, panicle length and \# tiller was collected over 5 fields with 11-day intervals from June to August. The fields, shown in Fig. 6c, were selected as a representative of the region in terms of agricultural practice diversity.

Ipsala agricultural site has been extensively analysed over the last years in the context of monitoring rice growth and mapping rice planting area with SAR images (Erten et al., 2016, 2015; Rossi and Erten, 2015; Yuzugullu et al., 2015). 


\begin{tabular}{lc}
\multicolumn{2}{c}{ Table 1: Parameters of TanDEM-X } \\
\hline Centre frequency & $9.65 \mathrm{GHz}$ \\
Bandwidth & $150 \mathrm{MHz}$ \\
Polarimetric channels & $\mathrm{HH}$ and VV \\
Azimuth resolution & $6.6 \mathrm{~m}$ \\
Pixel spacing in azimuth & $2.18-2.45 \mathrm{~m}$ \\
Slant-range resolution & $1.17 \mathrm{~m}$ \\
Pixel spacing in slant-range & $0.91 \mathrm{~m}$ \\
\hline
\end{tabular}

\subsection{TanDEM-X data}

The TanDEM-X data employed in this study were acquired during the science phase of this mission, from April to September 2015, and are provided in the Coregistered Single-Look Slant-range Complex (CoSSC) format, which is the standard for this data type and sensor. Each acquisition is composed by a pair of images: a monostatic image in which the same satellite is transmitter and receiver (which will be considered as master image), and a bistatic image in which the second satellite acts as a receiver (and which will be regarded as slave image). Both images are already coregistered in the standard CoSSC products. The main parameters of the TanDEM-X system and the resulting images are shown in Table 1.

The list of available acquisitions over each test site is included in Tables 2-4. There are 8 images in Sevilla and Ipsala, and only 6 in Valencia. The data in Sevilla presents the steepest incidence angle of the three datasets, approx. 23 degrees, in contrast to the 29 and 30 degrees of Valencia and Ipsala, respectively. The largest baselines are present also in the dataset of Sevilla, resulting in a height of ambiguity (HoA) around $2.5 \mathrm{~m}$. The HoA over Valencia is around $3.4 \mathrm{~m}$ and it is around $3.9 \mathrm{~m}$ in the Ipsala acquisitions (but for the last one with $5.09 \mathrm{~m}$ ). The extremely stable geometry of acquisition provided by this sensor along the whole campaign must be noted, which makes it very well suited for research and monitoring purposes. Regarding the acquisition time, the images over Sevilla were acquired at 06:30, the images over Valencia at 17:45, and the images over Ipsala at 04:30.

\subsection{SAR data processing}

Starting from the CoSSC product of each acquisition, the first step in the processing chain consists in the common range spectral filtering to compensate for the geometrical or baseline decorrelation $\gamma_{\text {geom }}$ cited in Section 2.1. This term is specially important for data acquired during the science phase of TanDEM-X, since the large baseline yields important shifts in the wavenumber, reaching values of $\gamma_{\text {geom }}$ below 0.8 .

After the range filtering we removed the flat Earth and topographic phase terms from the interferograms, 
Table 2: List of TanDEM-X image pairs over Sevilla

\begin{tabular}{cccccc}
\hline Date (yyyymmdd) & DoY & Master/Slave & Incidence angle (degrees) & $\kappa_{Z}(\mathrm{rad} / \mathrm{m})$ & HoA $(\mathrm{m})$ \\
\hline 20150604 & 155 & TDX/TSX & 22.71 & 2.48 & 2.53 \\
20150615 & 166 & TDX/TSX & 22.71 & 2.48 & 2.53 \\
20150626 & 177 & TDX/TSX & 22.73 & 2.48 & 2.53 \\
20150707 & 188 & TDX/TSX & 22.73 & 2.47 & 2.54 \\
20150718 & 199 & TDX/TSX & 22.73 & 2.48 & 2.53 \\
20150729 & 210 & TDX/TSX & 22.74 & 2.48 & 2.53 \\
20150809 & 221 & TDX/TSX & 22.73 & 2.49 & 2.52 \\
20150820 & 232 & TDX/TSX & 22.73 & 2.48 & 2.53 \\
\hline
\end{tabular}

Table 3: List of TanDEM-X image pairs over Valencia

\begin{tabular}{cccccc}
\hline Date (yyyymmdd) & DoY & Master/Slave & Incidence angle (degrees) & $\kappa_{Z}(\mathrm{rad} / \mathrm{m})$ & HoA $(\mathrm{m})$ \\
\hline 20150522 & 142 & TDX/TSX & 28.83 & 1.83 & 3.42 \\
20150602 & 153 & TDX/TSX & 28.84 & 1.83 & 3.43 \\
20150624 & 175 & TDX/TSX & 28.84 & 1.83 & 3.43 \\
20150705 & 186 & TDX/TSX & 28.83 & 1.84 & 3.41 \\
20150807 & 219 & TDX/TSX & 28.83 & 1.83 & 3.42 \\
20150818 & 230 & TDX/TSX & 28.83 & 1.84 & 3.42 \\
\hline
\end{tabular}

so the remaining interferometric phase only contains topographic information with respect to an arbitrary reference. When data are processed locally or at parcel level, one can also estimate locally the main fringe frequency and remove that contribution from the data. In our case, we assumed a constant topography over the processed subscene and computed the phase terms by exploiting the orbital information. Both approaches are valid for the PolInSAR algorithms applied afterwards, since all vegetation height estimations are carried out in relative terms. It must be noted that the absolute phase compensation employed by Rossi and Erten (2015) is not required. It is also important to mention that the use of external digital elevation models (DEM), such as the one provided by SRTM, may produce unwanted effects in this sort of scenes in which rice fields are located. The whole area of all three test sites is extremely flat, but the SRTM DEM exhibits a quantisation effect, with +/- $1 \mathrm{~m}$ jumps, which degrades importantly the quality of the interferometric phase and even its visual interpretation. Instead, we used arbitrary DEM's with constant values around the average height of each test site, i.e. $0 \mathrm{~m}$ for Valencia, $2 \mathrm{~m}$ for Sevilla, and $4 \mathrm{~m}$ for Ipsala.

Once the previous steps are carried out we formed the covariance/coherency matrices defined in (4-6) using a 21x21 boxcar speckle filter for multilooking. At this point we translated the polygons defining the 
Table 4: List of TanDEM-X image pairs over Ipsala

\begin{tabular}{cccccc}
\hline Date (yyyymmdd) & DoY & Master/Slave & Incidence angle (degrees) & $\kappa_{Z}(\mathrm{rad} / \mathrm{m})$ & HoA $(\mathrm{m})$ \\
\hline 20150606 & 157 & TDX/TSX & 29.99 & 1.61 & 3.89 \\
20150617 & 168 & TDX/TSX & 29.99 & 1.61 & 3.89 \\
20150709 & 190 & TDX/TSX & 29.99 & 1.62 & 3.89 \\
20150731 & 212 & TDX/TSX & 29.99 & 1.62 & 3.89 \\
20150811 & 223 & TDX/TSX & 29.99 & 1.62 & 3.88 \\
20150822 & 234 & TDX/TSX & 29.99 & 1.61 & 3.90 \\
20150902 & 245 & TDX/TSX & 29.99 & 1.61 & 3.89 \\
20150913 & 256 & TSX/TDX & 29.76 & 1.23 & 5.09 \\
\hline
\end{tabular}

regions of interest (ROI) of every parcel, originally in geographical coordinates, to radar coordinates (i.e. range and azimuth), so the ROIs of each parcel in the images could be analysed. An erosion with a kernel of size 11 was applied to the ROIs for avoiding the influence of the edges of the parcels, since the mentioned multilooking blurred the parcel borders and mixed the responses from outside the parcel with the inner parts. Alternatively, the multilooked data could have been geocoded or orthorectified and the subsequent processing done for pixels expressed in geographical coordinates.

Finally, the PolInSAR inversion algorithm was applied to each pixel inside the eroded ROI of each parcel. A single initial guess was employed in all cases: $h_{v}=1 \mathrm{~m}, \sigma=3 \mathrm{~dB} / \mathrm{m}, m_{D B \min }=-3 \mathrm{~dB}$, and $m_{D B \max }$ $=3 \mathrm{~dB}$. We run also some trials with different initial guesses but the resulting estimates, analysed at field scale, were very similar. Statistics of the obtained estimates within each field (histograms, average values and standard deviations) were computed and analysed, as it is shown in Section 4.

\section{Results}

In order to properly illustrate the methodology and the working principle of PolInSAR for rice height retrieval, this section starts by showing the appearance of the input data. Figure 7 shows the interferometric coherences and phases of the two copolar channels, for all dates, in the area in which a monitored field of the Sevilla site is located. Moreover, the border of the resulting regions of coherences (computed for the central pixel of the field) is displayed in the last column.

At the first date the coherences of both channels are very low, since plants have just emerged from the water surface and the backscattered power is very low (-19 $\mathrm{dB}$ at both linear channels), i.e. the flooded ground acts like a mirror. Consequently, the SNR decorrelation dominates both coherences. At the second date this situation changes dramatically and both coherences are very high. In this case the backscatter is 


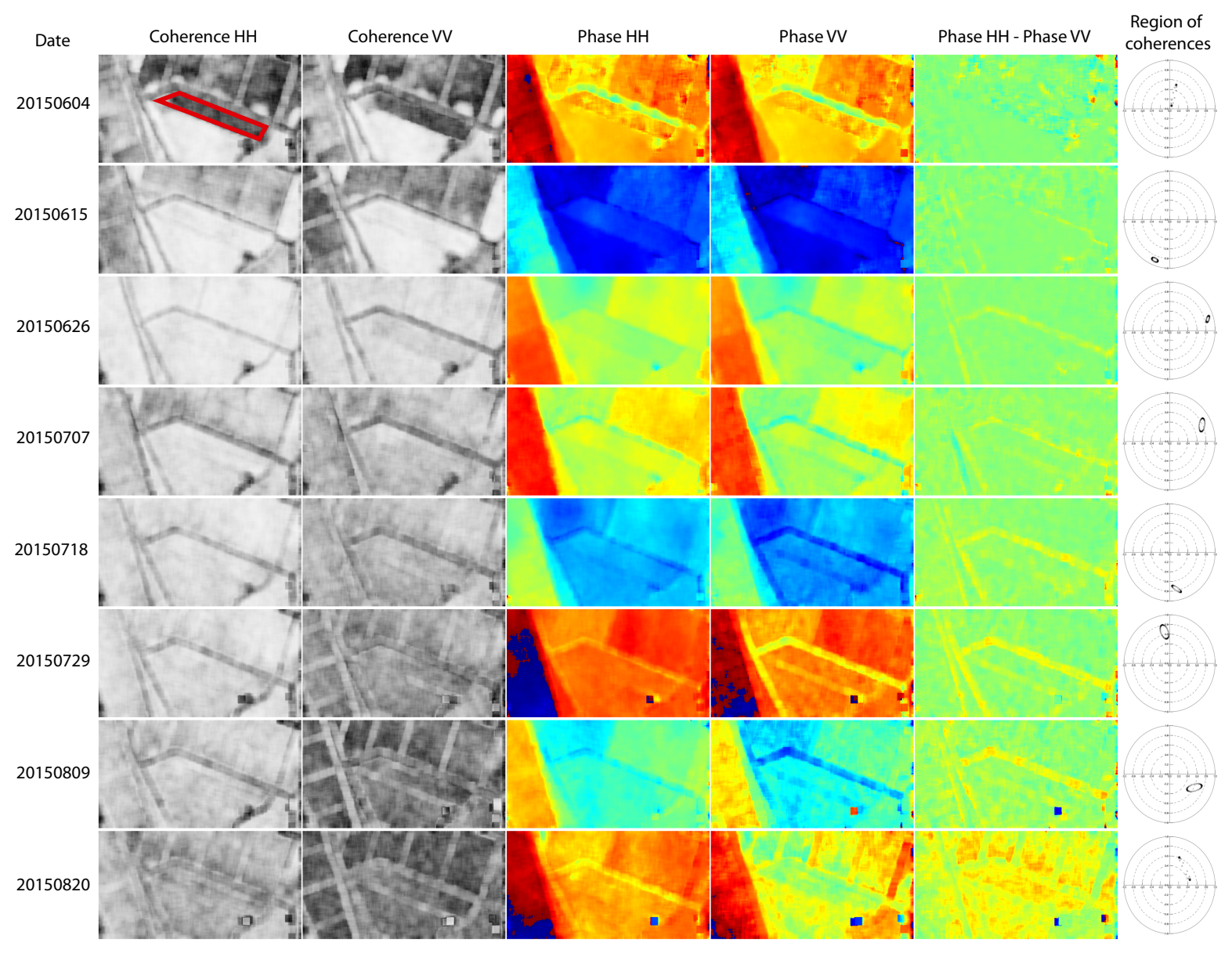

Figure 7: Maps of interferometric coherence, phase, and phase difference for the two copolar channels (HH and VV) in the area around field 1 of the Sevilla site, for all TanDEM-X acquisitions (8 dates). The field is marked with a red polygon in the first image. The last column shows the coherence regions obtained at the central pixel of the field.

much stronger (-7 dB at both linear channels) and can be interpreted as a direct surface contribution, since $\mathrm{HH}+\mathrm{VV}$ is very close to the total span and much higher than HH-VV (i.e. HH and VV are in phase). Taking into account the steep incidence angle (23 degrees) and the condition of the plants at this date (first stalks and tillers around $20 \mathrm{~cm}$ tall), it seems that the scattering corresponds to the one from a very rough surface, i.e. the ensemble of short plants and flooded ground act like a (very conducting) rough surface from the viewpoint of the radar. In addition, some water roughness is expected due to the presence of wind, which also contributes in the same way. This type of scattering mechanism produces a very localised radar response at ground level, hence the high coherence, but it also entails that all interferometric phases correspond to the same point along the vertical coordinate. This can be observed in the region of coherences, since it is very small and is close to the unit circumference. The lack of phase diversity at these early stages is the source of wrong height estimates, as will be discussed later in the text. 
From the third date onwards, the coherence at the $\mathrm{HH}$ channel decreases slowly, whereas the coherence at the VV channel exhibits a more pronounced decreasing trend. This is the same behaviour illustrated in Fig. 3, and is due to the lower backscattered power at VV than at $\mathrm{HH}$, widely studied in the literature (the $\mathrm{HH} / \mathrm{VV}$ ratio shows a peak, which is very characteristic of rice). In addition, as plants grow there start to appear a difference between the interferometric phases of the two copolar channels, which also translates into bigger coherence regions on the unit circle. These bigger coherence regions constitute a good sign for the performance of PolInSAR, since the retrieval of height is based on a necessary diversity in coherences and phases when observing the scene at different polarimetric channels.

Finally, it is important to clarify that the phases appearing in Fig. 7, and hence the position of the coherence region on the complex plane, can differ from date to date because no calibration with respect to a reference phase has been applied. Nonetheless, this does not affect the subsequent height retrieval, as it is mentioned in Section 2.2, since PolInSAR exploits only the relative phases between channels, not their absolute phases. Regarding the phase difference between HH and VV, shown also in Fig. 7, it is very small but increases with the development of the plants (as commented in the previous paragraph), reaching its maximum value, around 45 degrees, at the last date.

The following three figures, 8,9 and 11, present the temporal evolution of the height estimates at the three test sites, Sevilla, Valencia and Ipsala, respectively. In the case of Sevilla and Ipsala, each plot corresponds to a different field. Instead, we show only two plots for the Valencia site, each one corresponding to a different rice type and including all fields of that type.

The results obtained in Sevilla, shown in Fig. 8, exhibit two intervals with different performances. For all parcels the retrieved heights and the ground data are close from the third or fourth acquisition onwards (DoY $>170-180$ ), whereas before these dates the resulting heights are clearly overestimated and far from the actual values. In other words, the actual vegetation height must be above $25-30 \mathrm{~cm}$ to get accurate results. The first two or three acquisitions suffer an evident lack of interferometric sensitivity since for such short plants the spatial baselines should be even longer than the ones provided during the science phase of TanDEM-X. In the absence of sensitivity the retrieved heights are very noisy and strongly overestimated. Regarding the values obtained in the last 4 or 5 dates, at some fields there exits slight over- or underestimations, depending on field and date, but in general the correspondence is quite good. In addition, the variability inside the fields (indicated by the standard deviations) is small in terms of the vegetation heights.

The results obtained at the Valencia test site (Fig. 9) are somehow similar to the Sevilla site, but in this case there are only 6 acquisitions, so some intervals of the temporal evolution cannot be studied. For 

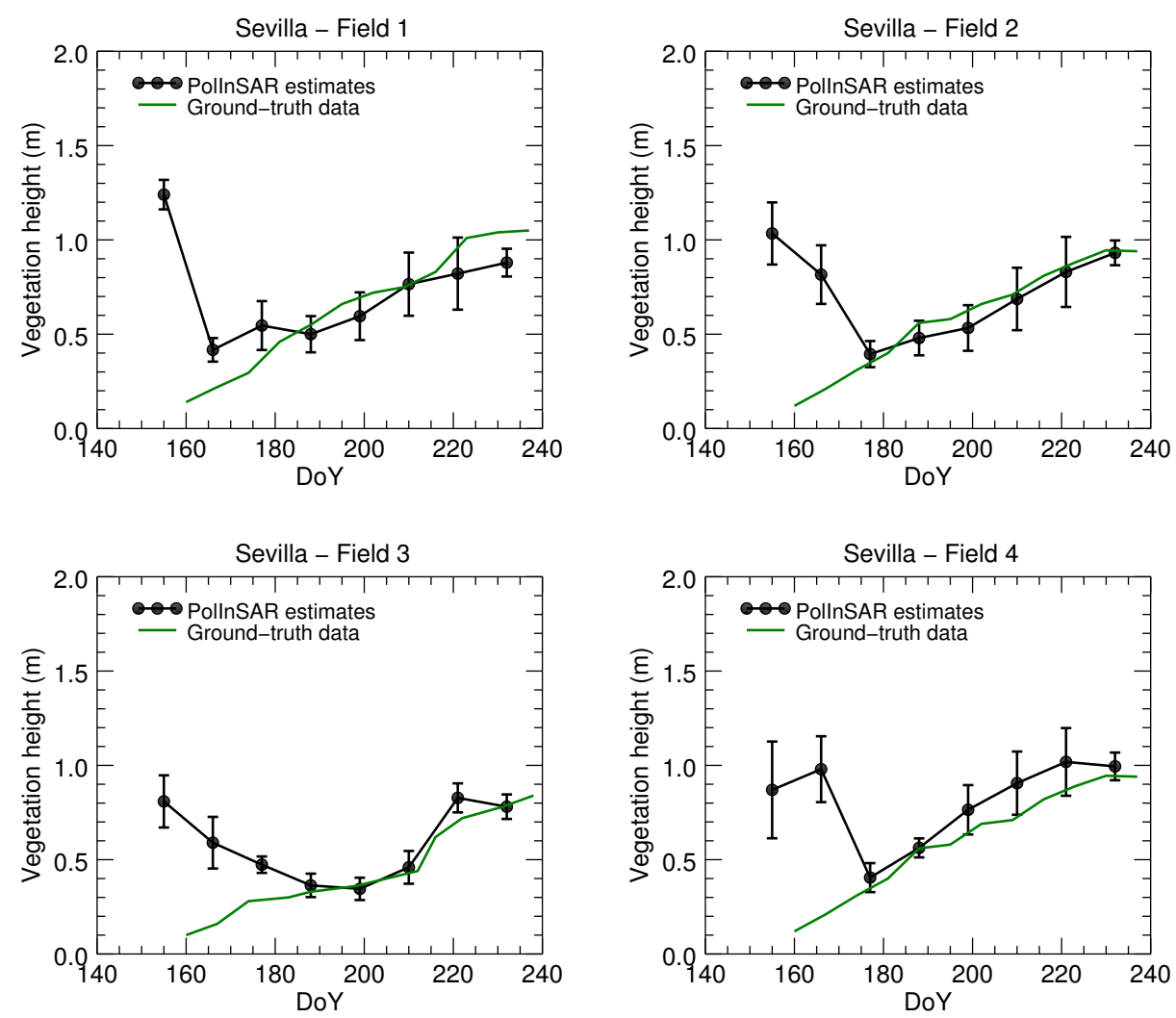

Figure 8: Temporal evolution of the vegetation height estimates and ground data for the four individual fields monitored in the Sevilla test site. Circles denote the average value and error bars denote $+/$ - one standard deviation, both computed for all pixels inside a field.

Senia rice type, only for the last two dates the estimates are close to the actual plant heights, whereas for Bomba rice the last three dates produce good results. As in the Sevilla case, it is clear that the plants have to exceed some height threshold for the technique to be applicable. In this case, the minimum required height is between 40 and $60 \mathrm{~cm}$, but it can not be defined better due to the lack of data in that interval. When compared to Sevilla, the smaller baseline, and hence the lower vertical sensitivity, justifies the requirement of a greater height threshold.

The results of the last three dates are shown for the individual fields in Fig. 9.c and 9.d, for Senia and Bomba rice types, respectively. For both rice types the standard deviations (i.e. intra-field variability) decrease consistently with time, being below $10 \mathrm{~cm}$ at the last date. At DoY 219 all heights for Senia rice are overestimated, but only in 3 fields this overestimation is greater than $10 \mathrm{~cm}$. Then, at the last date, the same 3 fields are the only ones to overestimate clearly the vegetation height, whereas the estimates for the other 13 fields are around the in situ data. Regarding the Bomba rice type, all 5 fields behave quite similarly, showing a significant underestimation only at DoY 219, whereas at the other dates the retrieved values are 


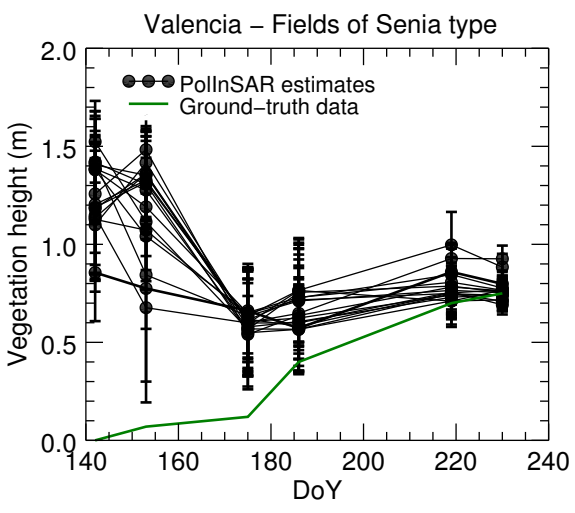

(a)

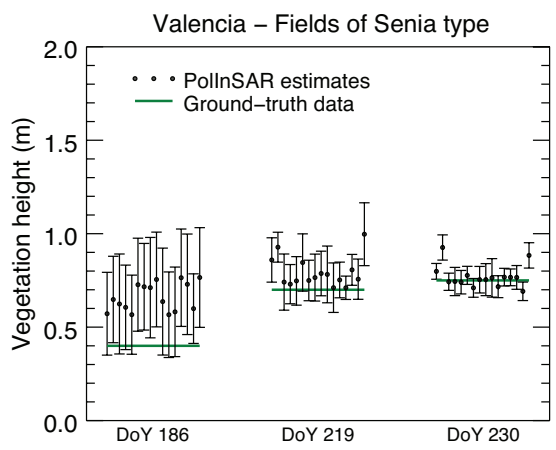

(c)

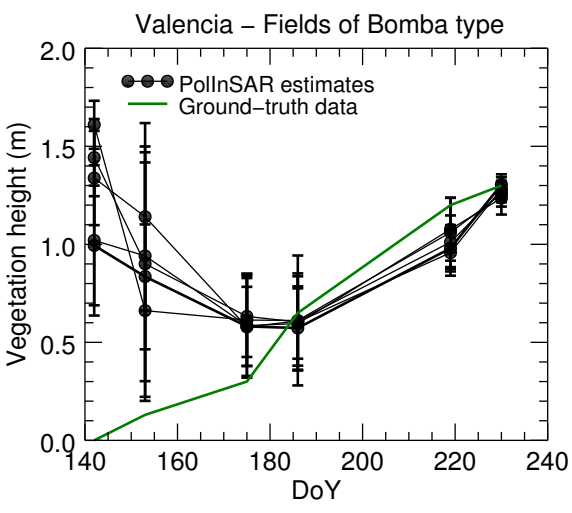

(b)

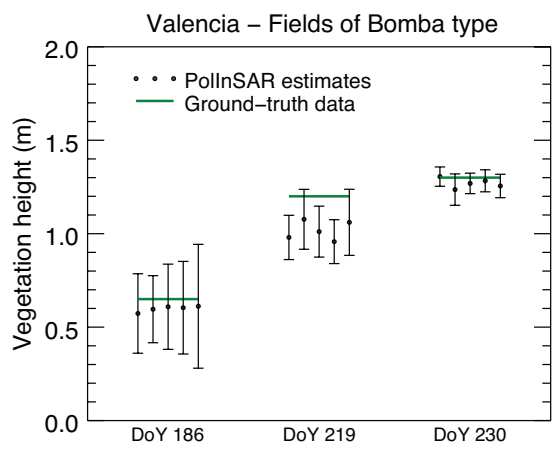

(d)

Figure 9: Top row: Temporal evolution of the vegetation height estimates and ground data for all the fields monitored in the Valencia test site, grouped by rice variety: (a) Senia, and (b) Bomba. Bottom row: Estimates obtained on the last three dates for each field separately, grouped by rice variety: (c) Senia, and (d) Bomba. Circles denote the average value and error bars denote one standard deviation, both computed for all pixels inside a field. Ground-truth (green line) is the average values of measured heights per variety and date, which showed a small variability (i.e. standard deviation values about $3 \mathrm{~cm}$ ) for both varieties.

around the ground-truth data.

For illustration purposes, Figure 10 shows an orthorectified image of the vegetation height estimates obtained in the rice fields of a large area of the Valencia test site on 18-Aug-2015 (i.e. on the last available acquisition date). One can easily distinguish the Bomba rice fields from the Senia rice ones thanks to their much greater height at this advanced stage. In addition, the fields of Bomba rice exhibit a very homogeneous height, whereas more variability can be found among the Senia rice fields present in the test site.

Finally, Figure 11 shows the results for the five fields monitored in Ipsala, which are similar to the ones obtained over Sevilla. Once again, we observe that from the third or fourth date onward (once vegetation is high enough) the retrieved values are in the same range as the validation data. The minimum height with accurate estimates is around $20-25 \mathrm{~cm}$. Unfortunately, for all fields except the first one, there is a clear 


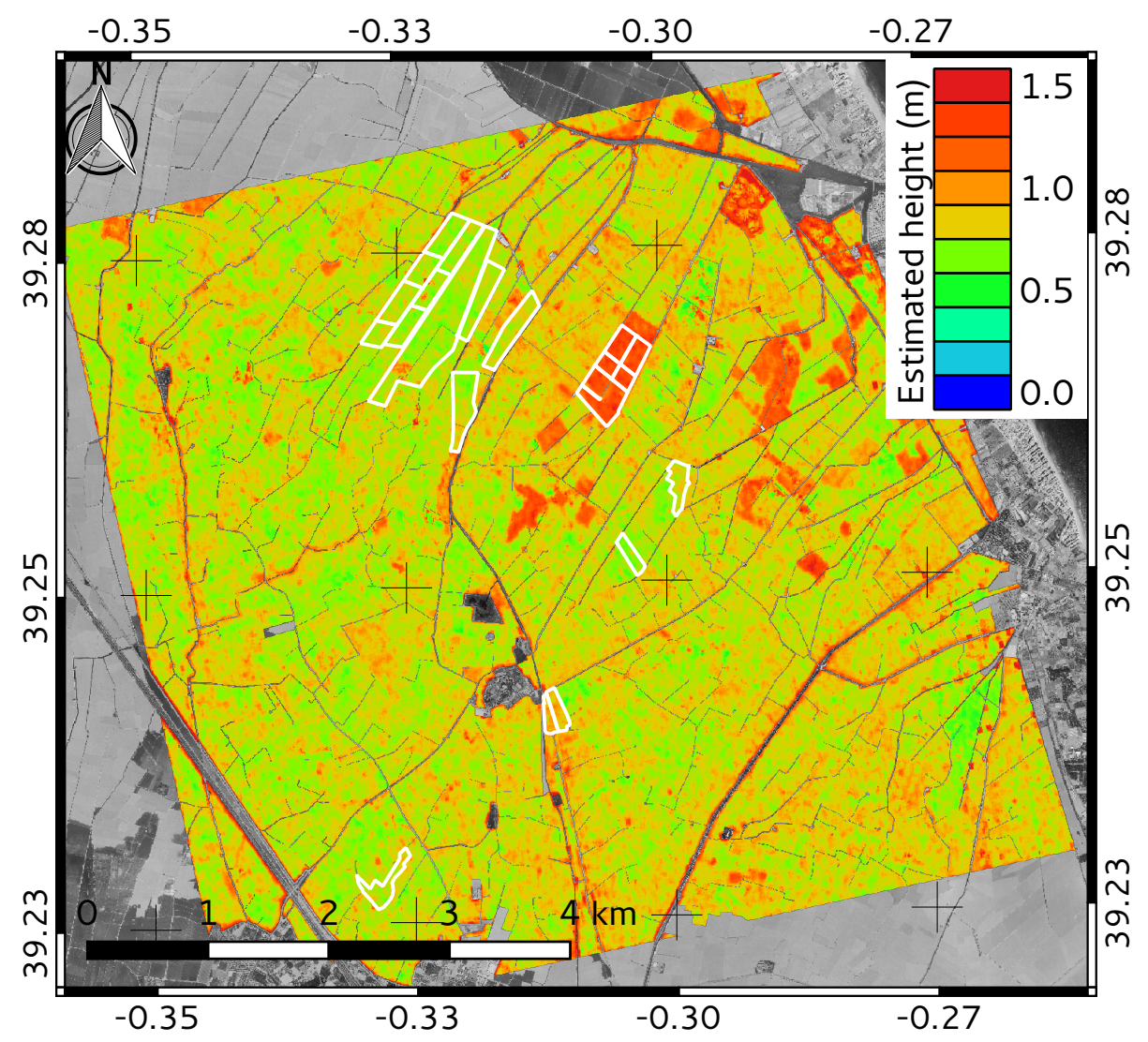

Figure 10: Vegetation height estimates obtained in the rice fields of a large area of the Valencia test site on 18-Aug-2015 (DoY 230), overlaid on a grey-scale orthophoto of the site. The borders of the monitored fields are shown in white colour.

underestimation of the height in the dates between DoY 220 and 250. Only for the last acquisition the estimates of all fields increase and, in some cases, are higher than the actual values. We have to remind that the last acquisition was gathered with a baseline smaller than the rest $(\mathrm{HoA}=5.09 \mathrm{~m}$ instead of $3.89 \mathrm{~m}$, see Table 4), so a worse performance was expected for the last date.

The justification of the resulting underestimation in Ipsala during the period with tall plants is not straightforward. At this point it is necessary to remind the main error sources outlined in Section 2.2.2, and in particular the potential mismatch between model and observations, and the influence of acquisition parameters (e.g. baseline and incidence angle). In order to know quantitatively the influence of these aspects on the performance of this technique, a comprehensive study on the potential and limitations of PolInSAR for rice height estimation should be carried out. Such an analysis requires the use of simulations for the main features of the scenes and the system with implications in PolInSAR, i.e. form of the coherency matrices of ground and vegetation, effect of available ground-to-volume ratios, presence of non-exponential scattering profiles, effect of differential extinction, influence of incidence angle and 

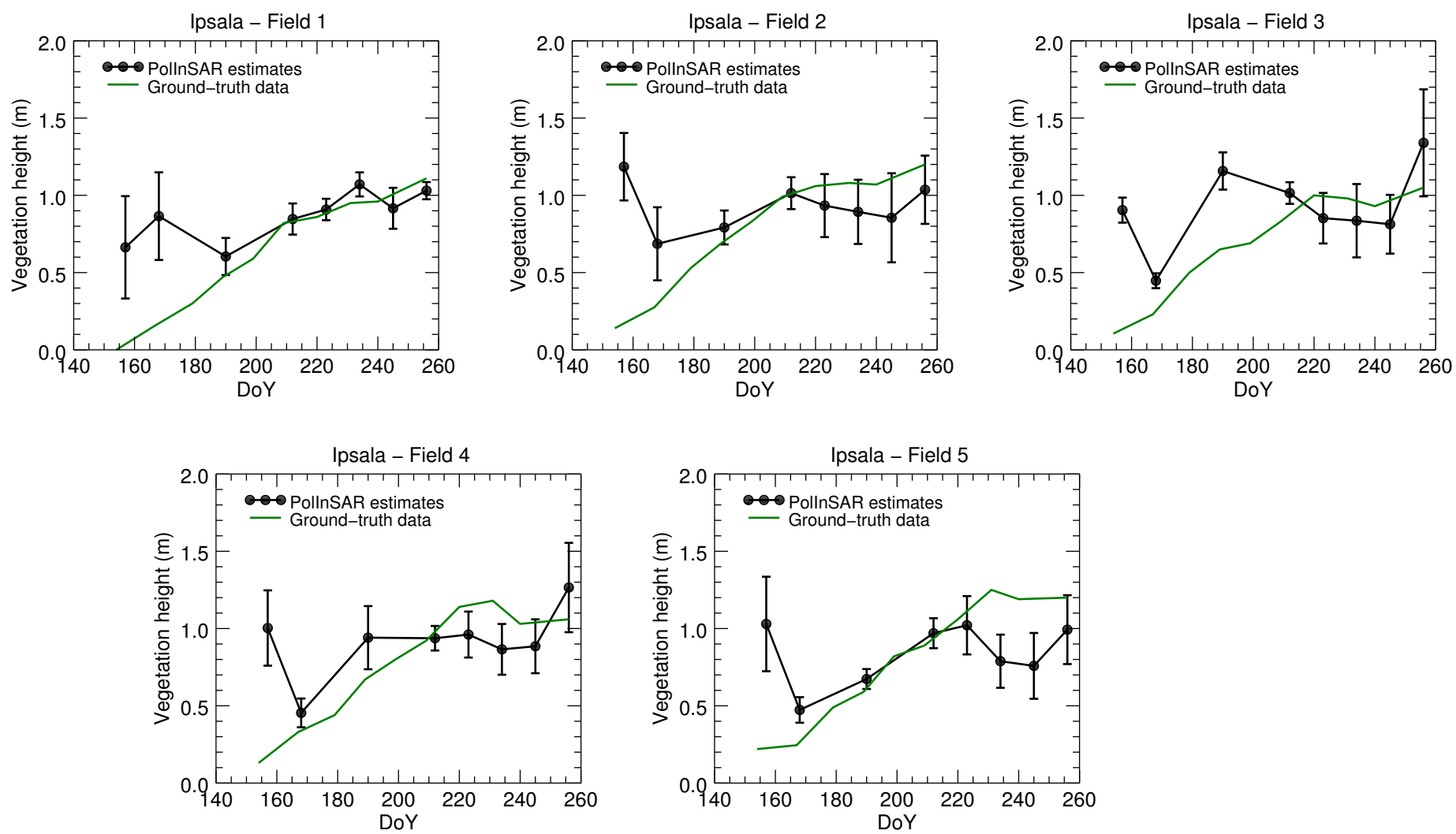

Figure 11: Temporal evolution of the vegetation height estimates and ground data for the five individual fields monitored in the Ipsala test site. Circles denote the average value and error bars denote one standard deviation, both computed for all pixels inside a field.

baseline, etc., following a procedure similar to that employed by Pichierri et al. (2016). This in-depth analysis has not been carried out for rice and single-transmit data yet, so it will be part of our future research on this topic. Besides simulations, a more complete validation with real data is also required, for which PolInSAR data acquired in additional configurations are needed. For instance, data gathered with the same incidence angle but with varying baseline, and vice versa, should be available over the same test site to ensure a proper comparison and to extract the separate and joint influence of each one of these two system parameters. In our case, we have worked with a single configuration for each test site and the baselines (i.e. heights of ambiguity) were different in all of them. Finally, for a really complete interpretation of all the mentioned aspects, in situ measurements should comprise also biomass, plant water content, and other crop-specific variables that help the physical characterisation of the scene.

A quantitative comparison between estimates and validation data for all test sites is illustrated in Figure 12. We show here the average values of retrieved heights per field and date, plotted against the data provided by the ground campaign. This representation confirms the previous comments regarding the 
Table 5: Statistics of the correlation between height estimates and validation data

\begin{tabular}{lccc}
\hline & Sevilla & Valencia & Ipsala \\
\hline$h_{v}$ threshold $(\mathrm{cm})$ & 25 & $40-60$ & 25 \\
$k_{v}$ threshold & 0.31 & $0.37-0.55$ & 0.20 \\
$R^{2}$ & 0.81 & 0.79 & 0.44 \\
RMSE $(\mathrm{cm})$ & 9.9 & 10.0 & 21.1 \\
Number of cases $(n)$ & 24 & 47 & 29 \\
\hline
\end{tabular}

height thresholds for a proper performance.

Following the discussion initiated in the Introduction about the necessity of large spatial baselines to provide enough sensitivity to the vertical distribution of scattering, some authors have suggested an optimum range of baselines which depends on the vegetation height to be retrieved and the rest of radar parameters (frequency and incidence angle) (Cloude, 2009; Lopez-Sanchez and Ballester-Berman, 2009; Pichierri, 2016). The best way to express this optimum range is by means of $k_{v}$, defined as the product of the vertical wavenumber $\kappa_{Z}$ and half of the vegetation height: $k_{v}=\kappa_{Z} h_{v} / 2$. This parameter is indeed the vertical coordinate employed in polarimetric coherence tomography (PCT) (Cloude, 2006, 2007), a technique derived from PolInSAR to estimate the vertical profile of scattering in vegetated scenes. Cloude (2009) showed that the best interval is $1 \leqslant k_{v} \leqslant 1.5$. Values lower than 1 do not guarantee enough volume decorrelation to be sensitive, whereas values higher than 1.5 compromise the coherence (it becomes very low) and hence its estimation itself.

According to the $\kappa_{Z}$ values shown in Tables $2-4$, a $k_{v}$ greater than 1 entails vegetation heights greater than $0.80 \mathrm{~m}, 1.08 \mathrm{~m}$ and $1.24 \mathrm{~m}$ for Sevilla, Valencia and Ipsala, respectively. Therefore our TanDEM-X data are out of the optimum range in most of the cases, since only the last dates in Sevilla and in Valencia (for Bomba rice) comply this criterion. The values of $k_{v}$ provided by the height thresholds at which the performance of PolInSAR starts to be satisfactory, attending to the analysis of Figures 8-11, are shown in Table 5, being all of them well below the suggested optimum range.

Table 5 also shows the determination coefficient $R^{2}$ and the root mean square error (RMSE) obtained at all test sites when considering only the range of estimates for which the ground data are above the mentioned thresholds. The number of cases $n$ is also shown in the table to interpret the statistical significance of these correlation indicators. We observe that $R^{2}$ is high and RMSE is small (above 0.7 and around $10 \mathrm{~cm}$, respectively) in Sevilla and Valencia. As for Ipsala, $R^{2}$ is lower than for the other two sites $(0.44)$ and the corresponding RMSE has doubled $(21 \mathrm{~cm})$. 

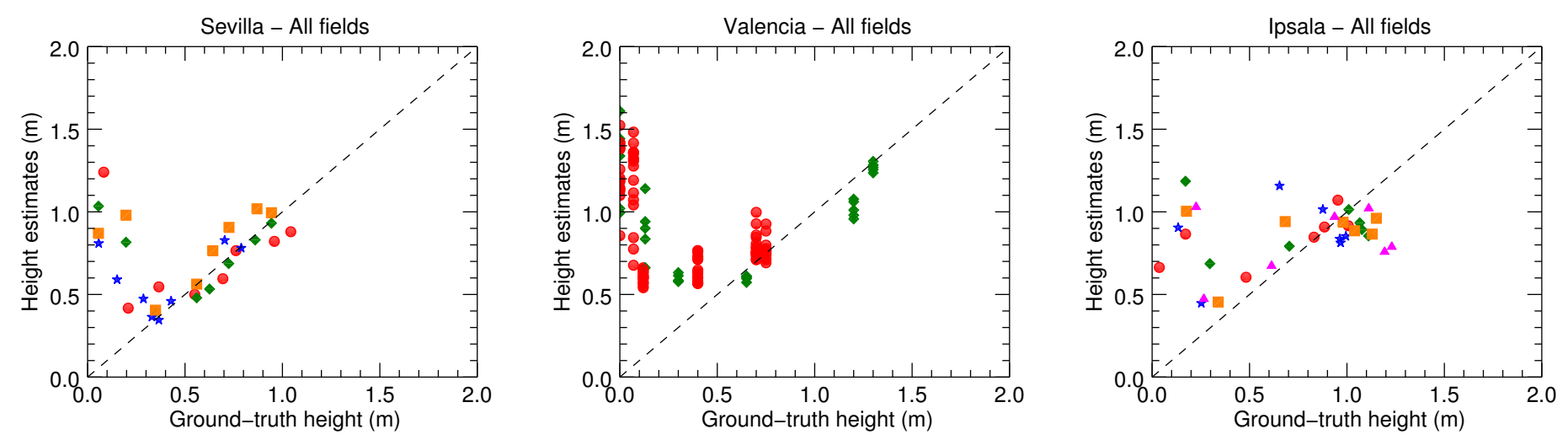

Figure 12: Comparison of the average height estimates per field against the ground data for the three test sites (Sevilla, Valencia and Ipsala). Different symbols correspond to different fields in Sevilla and Ipsala, and to different rice varieties in Valencia. The last acquisition in Ipsala is excluded due to its larger baseline.

\section{Conclusions}

The work presented here constitutes the first complete demonstration of crop height retrieval based on PolInSAR with satellite data. This technique has been tested with rice fields located in three different sites, covering the whole growth season. The proposed methodology is fully automatic without relying on external reference information. Besides the novelty of the source of data, a modified inversion algorithm has been proposed, which is especially adapted to scenes in which the main ground contribution is the double-bounce between stalks and ground, as it happens in rice fields when the ground is flooded, and is suitable for data acquired in single-transmit mode (also known as bistatic mode in the TanDEM-X mission).

The proposed methodology is useful to monitor the development of rice fields, since satisfactory results have been obtained in all test sites, with RMSE from 10 to $20 \mathrm{~cm}$. The main limitation appears at the early cultivation dates because the available spatial baselines are not large enough to monitor very short vegetation, even the ones provided during the science phase of TanDEM-X. For each test site a height threshold for right performance has been found. The actual value of this threshold depends on the baseline, the incidence angle and the scene properties, since not all rice types present the same radar and interferometric pattern.

The retrieved height values have shown to capture the seasonal rice growth variations present along the cultivation cycle. In addition, estimated plant height allows discriminating between varieties with distinctive height and morphology, such as Bomba and Senia rice in the Valencia test site.

This research will continue with an analysis and interpretation of the estimates of the rest of model parameters (extinction and ground-to-volume ratios). The potential use of an external DEM, obtained when there is no vegetation on the field, employed as a topographic reference to help PolInSAR, could improve 
the estimation performance. A comprehensive study on the potential and limitations of PolInSAR for rice height estimation will be carried out by exploiting simulations to test the influence of the main features of the scenes and the system configuration, and assessing all theoretical and numerical aspects involved in this approach. Finally, the same general approach has to be assessed with other agriculture landscapes, by adapting the formulation and the inversion procedure to crops with non-flooded ground conditions.

\section{Acknowledgement}

The German Aerospace Center (DLR) provided all the TanDEM-X data under project NTI-POLI6736. The authors would like to thank the support of the Federacion de Arroceros de Sevilla, for providing the ground measurement data and for their helpful comments. The orthorectified photographs over Sevilla and Valencia sites were provided by the PNOA program of the Instituto Geográfico Nacional (IGN) 2016.

This work has been supported by the Spanish Ministry of Economy and Competitiveness (MINECO) and EU FEDER under project TIN2014-55413-C2-2-P. The research leading to these results has received funding from the European Union Seventh Framework Programme (FP7/2007-2013) under grant agreement 606983, and the Land-SAF (the EUMETSAT Network of Satellite Application Facilities) project. The insitu measurements in the Ipsala site were conducted with the funding of The Scientific and Technological Research Council of Turkey (TUBITAK, Project No:113Y446).

\section{References}

Abdullahi S, Kugler F, Pretzsch H. Prediction of stem volume in complex temperate forest stands using TanDEM-X SAR data. Remote Sensing of Environment 2016;174:197-211. doi:10.1016/j.rse. 2015.12 .012 .

Ballester-Berman JD, Lopez-Sanchez JM. Coherence loci for a homogeneous volume over a double-bounce ground return. IEEE Geosci Remote Sensing Letters 2007;4(2):317-21. doi:10 .1109/LGRS . 2007. 894161.

Ballester-Berman JD, Lopez-Sanchez JM. Combination of direct and double-bounce ground responses in the homogeneous oriented volume over ground model. IEEE Geosci Remote Sensing Letters 2011;8(1):54-8. doi:10.1109/LGRS.2010.2051016.

Ballester-Berman JD, Lopez-Sanchez JM, Fortuny-Guasch J. Retrieval of biophysical parameters of agricultural crops using polarimetric SAR interferometry. IEEE Trans Geosci Remote Sensing 2005;43(4):683-94. doi:10.1109/TGRS . 2005 . 843958. 
Bamler R, Hartl P. Synthetic aperture radar interferometry. Inverse Problems 1998;14(4):R1. doi:10 . $1088 / 0266-5611 / 14 / 4 / 001$.

Campos-Taberner M, García-Haro FJ, Moreno A, Gilabert MA, Sánchez-Ruiz S, Martínez B, Camps-Valls G. Mapping leaf area index with a smartphone and Gaussian processes. IEEE Geosci Remote Sensing Letters 2015;12(12):2501-5. doi:10.1109/LGRS . 2015.2488682.

Campos-Taberner M, Garcia-Haro FJ, Camps-Valls G, Grau-Muedra G, Nutini F, Crema A, Boschetti M. Multitemporal and multiresolution leaf area index retrieval for operational local rice crop monitoring. Remote Sensing of Environment 2016a;187:102-18. doi:10.1016/j.rse.2016.10.009.

Campos-Taberner M, Garcia-Haro FJ, Confalonieri R, Martinez B, Moreno A, Sanchez-Ruiz S, Gilabert MA, Camacho F, Boschetti M, Busetto L. Multitemporal monitoring of plant area index in the Valencia rice district with PocketLAI. Remote Sensing 2016b;8:202. doi:10 .3390/rs 8030202.

Cloude SR. Polarization coherence tomography. Radio Science 2006;41(4). doi:10.1029/ 2005 RS 003436.

Cloude SR. Dual-baseline coherence tomography. IEEE Geosci Remote Sensing Letters 2007;4(1):127-31. doi:10.1109/LGRS.2006.885893.

Cloude SR. Polarisation: applications in remote sensing. Oxford University Press, 2009.

Cloude SR, Papathanassiou KP. Polarimetric SAR interferometry. IEEE Trans Geosci Remote Sensing 1998;36(5):1551-65. doi:10.1109/36.718859.

Cloude SR, Papathanassiou KP. Three-stage inversion process for polarimetric SAR interferometry. IEE Proc-Radar, Sonar Navig 2003;150(3):125-34. doi:10.1049/ip-rsn:20030449.

Erten E, Lopez-Sanchez JM, Yuzugullu O, Hajnsek I. Retrieval of agricultural crop height from space: A comparison of SAR techniques. Remote Sensing of Environment 2016;187:130-44. doi:10.1016/j . rse.2016.10.007.

Erten E, Rossi C, Yuzugullu O. Polarization impact in TanDEM-X data over vertical-oriented vegetation: the paddy-rice case study. IEEE Geosci Remote Sensing Letters 2015;12(7):1501-5. doi:10.1109/ LGRS.2015.2410339.

Ferro-Famil L, Neumann M, Huang Y. Multi-baseline POL-inSAR statistical techniques for the characterization of distributed media. In: Proceedings of the IEEE International Geoscience and Remote 
Sensing Symposium (IGARSS). Cape Town, South Africa; volume 3; 2009. p. 971-4. doi:10 .1109/ IGARSS.2009.5417937.

Flynn T, Tabb M, Carande R. Coherence region shape extraction for vegetation parameter estimation in polarimetric SAR interferometry. In: Proceedings of the IEEE International Geoscience and Remote Sensing Symposium (IGARSS). Toronto, Canada; volume 5; 2002. p. 2596-8. doi:10 . 1109 / IGARSS . 2002.1026712

Garestier F, Dubois-Fernandez PC, Papathanassiou KP. Pine forest height inversion using single-pass Xband PolInSAR data. IEEE Trans Geosci Remote Sensing 2008;46(1):59-68. doi:10 .1109/TGRS . 2007.907602.

Gatelli F, Monti Guarnieri A, Parizzi F, Pasquali P, Prati C, Rocca F. The wavenumber shift in SAR interferometry. IEEE Trans Geosci Remote Sensing 1994;32(4):855-64. doi:10.1109/36.298013.

Gomez-Dans JL, Quegan S, Bennett JC. Indoor C-band polarimetric interferometry observations of a mature wheat canopy. IEEE Trans Geosci Remote Sensing 2006;44(4):768-77. doi:10.1109/TGRS .2005. 863861.

Hajnsek I, Kugler F, Lee SK, Papathanassiou KP. Tropical-forest-parameter estimation by means of PolInSAR: The INDREX-II campaign. IEEE Trans Geosci Remote Sensing 2009;47(2):481-93. doi:10 . $1109 /$ TGRS. 2008.2009437.

Krieger G, Moreira A, Fiedler H, Hajnsek I, Werner M, Younis M, Zink M. TanDEM-X: A satellite formation for high-resolution SAR interferometry. IEEE Trans Geosci Remote Sensing 2007;45(11):3317-41. doi:10.1109/TGRS.2007.900693.

Kucuk C, Taskin G, Erten E. Paddy-rice phenology classification based on machine-learning methods using multitemporal co-polar X-band SAR images. IEEE J Sel Topics Appl Earth Observ Remote Sens 2016;9(6):2509-19. doi:10.1109/JSTARS . 2016.2547843.

Kugler F, Lee SK, Hajnsek I, Papathanassiou KP. Forest height estimation by means of Pol-InSAR data inversion: The role of the vertical wavenumber. IEEE Trans Geosci Remote Sensing 2015;53(10):5294311. doi:10.1109/TGRS.2015.2420996.

Kugler F, Schulze D, Hajnsek I, Pretzsch H, Papathanassiou KP. TanDEM-X Pol-InSAR performance for forest height estimation. IEEE Trans Geosci Remote Sensing 2014;52(10):6404-22. doi:10.1109/ TGRS.2013.2296533. 
Lee SK, Fatoyinbo TE. TanDEM-X Pol-InSAR inversion for mangrove canopy height estimation. IEEE J Sel Topics Appl Earth Observ Remote Sens 2015;8(7):3608-18. doi:10.1109/jstars.2015 . 2431646 .

Lee SK, Kugler F, Papathanassiou KP, Hajnsek I. Quantification of temporal decorrelation effects at Lband for polarimetric SAR interferometry applications. IEEE J Sel Topics Appl Earth Observ Remote Sens 2013;6(3):1351-67. doi:10.1109/JSTARS . 2013.2253448.

Lopez-Sanchez JM, Ballester-Berman JD. Potentials of polarimetric SAR interferometry for agriculture monitoring. Radio Science 2009;44(2). doi:10.1029/2008RS 004078.

Lopez-Sanchez JM, Ballester-Berman JD, Hajnsek I. First results of rice monitoring practices in Spain by means of time series of TerraSAR-X dual-pol images. IEEE J Sel Topics Appl Earth Observ Remote Sens 2011;4(2):412-22. doi:10 .1109/JSTARS . 2010.2047634.

Lopez-Sanchez JM, Ballester-Berman JD, Marquez-Moreno Y. Model limitations and parameter estimation methods for agricultural applications of polarimetric SAR interferometry. IEEE Trans Geosci Remote Sensing 2007;45(11):3481-93. doi:10.1109/TGRS.2007.900690.

Lopez-Sanchez JM, Cloude SR, Ballester-Berman JD. Rice phenology monitoring by means of SAR polarimetry at X-band. IEEE Trans Geosci Remote Sensing 2012a;50(7):2695-709. doi:10.1109/ TGRS.2011.2176740.

Lopez-Sanchez JM, Hajnsek I, Ballester-Berman JD. First demonstration of agriculture height retrieval with PolInSAR airborne data. IEEE Geosci Remote Sensing Letters 2012b;9(2):242-6. doi:10.1109/ LGRS . 2011.2165272.

Lopez-Sanchez JM, Vicente-Guijalba F, Ballester-Berman JD. The problem of SNR in PolInSAR observations with TanDEM-X over rice fields. In: Proceedings of the 4th TanDEM-X Science Team Meeting. DLR, Wessling, Germany; 2013.

Lopez-Sanchez JM, Vicente-Guijalba F, Ballester-Berman JD, Cloude SR. Polarimetric response of rice fields at C-band: Analysis and phenology retrieval. IEEE Trans Geosci Remote Sensing 2014;52(5):2977-93. doi:10 .1109/TGRS . 2013.2268319.

Lopez-Sanchez JM, Vicente-Guijalba F, Ballester-Berman JD, Cloude SR. Influence of incidence angle on the coherent copolar polarimetric response of rice at X-band. IEEE Geosci Remote Sensing Letters 2015;12(2):249-53. doi:10 .1109/LGRS . 2014 . 2334371. 
Martone M, Brautigam B, Krieger G. Quantization effects in TanDEM-X data. IEEE Trans Geosci Remote Sensing 2015;53(2):583-97. doi:10 .1109/TGRS .2014 .2325976.

Papathanassiou KP, Cloude SR. Single baseline polarimetric SAR interferometry. IEEE Trans Geosci Remote Sensing 2001;39(11):2352-63. doi:10.1109/36.964971.

Pichierri M. Multi-baseline Polarimetric SAR Interferometry for Characterizing the Biophysical Properties of Agricultural Crops. Ph.D. thesis; ETH Zurich; 2016.

Pichierri M, Hajnsek I, Papathanassiou KP. A multibaseline Pol-InSAR inversion scheme for crop parameter estimation at different frequencies. IEEE Trans Geosci Remote Sensing 2016;54:4952-70. doi:10 . 1109 /TGRS.2016.2553739.

Praks J, Kugler F, Papathanassiou KP, Hajnsek I, Hallikainen M. Height estimation of boreal forest: Interferometric model-based inversion at L- and X-band versus HUTSCAT profiling scatterometer. IEEE Geosci Remote Sensing Letters 2007;4(3):466-70. doi:10.1109/LGRS . 2007 . 898083.

Rossi C, Erten E. Paddy-rice monitoring using TanDEM-X. IEEE Trans Geosci Remote Sensing 2015;53(2):900-10. doi:10.1109/TGRS.2014.2330377.

Sagués L, Lopez-Sanchez JM, Fortuny J, Fàbregas X, Broquetas A, Sieber AJ. Indoor experiments on polarimetric SAR interferometry. IEEE Trans Geosci Remote Sensing 2000;38(2):671-84. doi:10 . $1109 / 36.841997$.

Treuhaft RN, Madsen SN, Moghaddam M, van Zyl JJ. Vegetation characteristics and underlying topography from interferometric radar. Radio Science 1996;31(6):1449-85. doi:10 .1029/96RS 01763.

Treuhaft RN, Siqueira PR. Vertical structure of vegetated land surfaces from interferometric and polarimetric data. Radio Science 2000;35(1):141-77. doi:10.1029/1999RS900108.

Yuzugullu O, Erten E, Hajnsek I. Rice growth monitoring by means of X-band co-polar SAR: Feature clustering and BBCH scale. IEEE Geosci Remote Sensing Letters 2015;12(6):1218-22. doi:10 .1109/ LGRS. 2015.2388953. 


\section{List of Figures}

1 Unit circle on the complex plane with the coherence region (grey ellipse) and the coherences with maximum ground contribution $\gamma\left(\kappa_{Z}, \vec{w}_{\max }\right)$ (brown point) and with minimum ground contribution $\gamma\left(\kappa_{Z}, \vec{w}_{\min }\right)$ (green point). The line corresponds to the standard RVoG model, which crosses the unit circumference at the topographic phase $\phi_{0} \ldots \ldots \ldots \ldots$

2 Flow diagram of the proposed inversion algorithm for PolInSAR data and scenes with a dominant double-bounce ground contribution. . . . . . . . . . . . 9

3 Comparison of the total measured coherence (after range spectral filtering) and the SNR decorrelation term, $\gamma_{\mathrm{SNR}}$, for the two copolar channels obtained for a rice field in Sevilla during the 2015 campaign. Left: HH. Right VV. . . . . . . . . . . . . . . . . . . . 11

4 Unit circle on the complex plane with the coherences and the line corresponding to the RVoG model in equation (11). $\gamma_{D B}$ is the decorrelation term due to the dominance of the doublebounce contribution at the ground. The true topographic phase $\phi_{0}$ is defined by the crossing of the line with the circumference of radius $\gamma_{D B}$, which is different from the phase $\phi_{0}^{\prime}$ that would have been obtained by the crossing with the unit circumference. . . . . . . . . . . 13

5 Numerical assessment of the inversion algorithm: vegetation height estimates (mean and

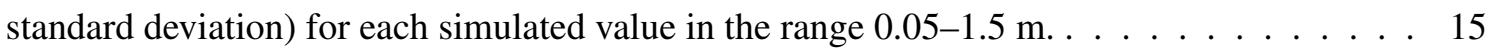

6 Location and orthophotos of the 3 test sites (Sevilla, Valencia and Ipsala). All fields monitored in 2015 are highlighted. In the case of Valencia, the fields coloured in red correspond to Senia type and fields coloured in yellow correspond to Bomba type. . . . . . . 16

7 Maps of interferometric coherence, phase, and phase difference for the two copolar channels (HH and VV) in the area around field 1 of the Sevilla site, for all TanDEM-X acquisitions ( 8 dates). The field is marked with a red polygon in the first image. The last column shows the coherence regions obtained at the central pixel of the field. . . . . . . . . . . 21

8 Temporal evolution of the vegetation height estimates and ground data for the four individual fields monitored in the Sevilla test site. Circles denote the average value and error bars denote $+/$ - one standard deviation, both computed for all pixels inside a field. . . . . . . . 23 
9 Top row: Temporal evolution of the vegetation height estimates and ground data for all the fields monitored in the Valencia test site, grouped by rice variety: (a) Senia, and (b) Bomba. Bottom row: Estimates obtained on the last three dates for each field separately, grouped by rice variety: (c) Senia, and (d) Bomba. Circles denote the average value and error bars denote one standard deviation, both computed for all pixels inside a field. Ground-truth (green line) is the average values of measured heights per variety and date, which showed a small variability (i.e. standard deviation values about $3 \mathrm{~cm}$ ) for both varieties. . . . . . . . . 24

10 Vegetation height estimates obtained in the rice fields of a large area of the Valencia test site on 18-Aug-2015 (DoY 230), overlaid on a grey-scale orthophoto of the site. The borders of the monitored fields are shown in white colour. . . . . . . . . . . . . . . . . . 25

11 Temporal evolution of the vegetation height estimates and ground data for the five individual fields monitored in the Ipsala test site. Circles denote the average value and error bars denote one standard deviation, both computed for all pixels inside a field. . . . . . . . . . . . 26

12 Comparison of the average height estimates per field against the ground data for the three test sites (Sevilla, Valencia and Ipsala). Different symbols correspond to different fields in Sevilla and Ipsala, and to different rice varieties in Valencia. The last acquisition in Ipsala is

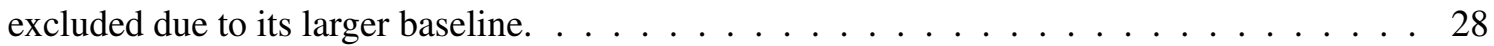

